\title{
Chacé, les Rogelins : un site des débuts de La Tène en Anjou
}

Chacé, les Rogelins : a site from the beginning of La Tène in Anjou

Chacé, les Rogelins: ein Fundplatz vom Beginn der Latènezeit im Anjou

Olivier Nillesse, Rémy Arthuis, Anna Baudry, Frédérique Durand, Elisabeth

Rousseau et Carole Vissac

\section{(2) OpenEdition}

Journals

Édition électronique

URL : https://journals.openedition.org/rao/3946

DOI : $10.4000 /$ rao.3946

ISSN : $1775-3732$

Éditeur

Presses universitaires de Rennes

Édition imprimée

Date de publication : 31 décembre 2017

Pagination : 143-176

ISBN : 978-2-7535-7540-0

ISSN : 0767-709X

\section{Référence électronique}

Olivier Nillesse, Rémy Arthuis, Anna Baudry, Frédérique Durand, Elisabeth Rousseau et Carole Vissac,

"Chacé, les Rogelins : un site des débuts de La Tène en Anjou », Revue archéologique de l'Ouest [En

ligne], 34 | 2017, mis en ligne le 13 juin 2020, consulté le 22 août 2022. URL : http://

journals.openedition.org/rao/3946; DOI : https://doi.org/10.4000/rao.3946 


\title{
Chacé, les Rogelins : un site des débuts de La Tène en Anjou
}

\author{
Chacé, les Rogelins: A Site from the Beginning of La Tène in Anjou
}

\author{
Olivier Nillesse ${ }^{a}$, Rémy Arthuis ${ }^{b}$, Anna BaudrY $^{c}$, Frédérique Durand ${ }^{d}$, \\ Elisabeth Rousseau ${ }^{\mathrm{e}}$ et Carole VISSAC ${ }^{\mathrm{f}}$
}

\begin{abstract}
Résumé : La fouille menée par l'INRAP à Chacé dans le Maine-et-Loire a permis d'étudier une partie d'un habitat du second Âge du Fer caractérisée par des silos. L'étude du mobilier indique une occupation de La Tène ancienne, période mal documentée dans la région des Pays de la Loire. Des analyses géomorphologiques, micromorphologiques, l'examen des restes osseux animaux et humains et des carporestes complètent l'interprétation du site.

Abstract: The excavation led by INRAP has allowed us to study a part of a settlement dating to the Late Iron Age characterized by storage pits. The study of the artefacts indicates an occupation of the Early La Tène, a period little documented in the region of the Pays de la Loire. Geomorphological, micromorphological analyses, the examination of human and animal bones and carpology enhance the site's interpretation.
\end{abstract}

Mots clés : La Tène ancienne, habitat ouvert, silos, céramique, analyses.

Keywords: Early La Tène, open settlement, storage pit, ceramic, analysis.

\section{INTRODUCTION}

La fouille réalisée à Chacé dans le Maine-et-Loire (fig. 1) sur une superficie de $26500 \mathrm{~m}^{2}$ a permis de mettre au jour deux occupations distinctes. Seule la première attribuée à La Tène A2/B1 fait l'objet de cet article, la seconde beaucoup moins documentée appartient au début du Haut-Empire (fig. 2).

\section{ConteXte géographiQue ET GÉOMORPHOLOGIQUE}

La commune de Chacé est située sur un plateau limité à l'ouest par l'incision brutale de la vallée du Thouet et dominé à l'est par les collines de Varrains. La parcelle étudiée présente une pente nord-sud de $2 \%$ qui s'accentue légèrement à la cote 35,5 m NGF (fig. 3). Cette rupture de pente est soulignée par le cadastre du XIx ${ }^{e}$ siècle qui présente au nord, un parcellaire laniéré orienté est-ouest et au sud, un second système orienté nord-sud.

\footnotetext{
a INRAP Grand Ouest/UMR 6566 CReAAH - 3 route de Damvix, Bazoin, 85420 DAMVIX.

b INRAP Grand Ouest/LARA-UMR 6566 CReAAH. Centre archéologique de Carquefou, 4 rue du Tertre, 44470 CARQUEFOU cedex.

' INRAP Grand Sud-Ouest/UMR 6566 CReAAH - 122 rue de la Bugellerie, 86000 POITIERS.

'INRAP GSO/UMR 5608 Traces. Maison de la recherche - 5 allée A.-Machado, 31058 TOULOUSE cedex 9.

e 16 rue Eugène-Cornet, 44100 NANTES.

${ }^{\mathrm{f}}$ GéoArchEon. 30, rue de la Victoire, 55210 VIÉVILLE-SOUS-LES-CÔTES.
} 


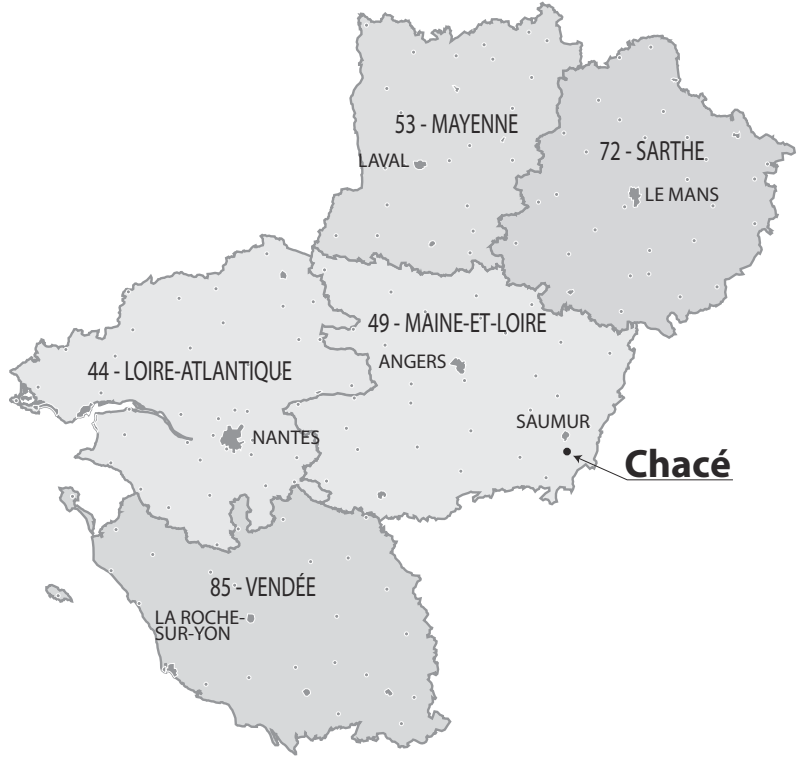

Figure 1 : Localisation de la commune de Chacé dans le Maineet-Loire. DAO J.-M. Bryand.

Figure 1: Location of the municipality of Chace in Maine-et-Loire.
Le substratum révélé par le décapage des terres végétales est composé des craies blanches du Turonien inférieur (C3a) plus ou moins désagrégées. Dans la zone de rupture de pente, la craie est recouverte d'un niveau de fines argileuses et d'amas de craie blanche de couleur orangée, correspondant à un horizon d'accumulation d'argile (niveau 2, $\operatorname{logs} 1$ et 2 : fig. 3). Au-dessus, les sédiments observés correspondent à des niveaux transformés ou remaniés par l'activité humaine.

Lobservation des sédiments en coupe montre une couverture sédimentaire qui s'accentue en direction du sud jusqu'à la rupture de pente, avec, au nord, un substratum crayeux tronqué, et au sud, la conservation sur le substratum crayeux d'un horizon d'accumulation d'argile, lui-même recouvert d'un sol anthropisé et de plusieurs niveaux de colluvions (niveaux 3, 6 et 7, logs 1 et 2 : fig. 3). Au sud de la rupture de pente, ce sol est tronqué, et les niveaux colluvionnés disparaissent rapidement.

Cette configuration sédimentaire reflète une distribution acquise entre la mise place du parcellaire et aujourd'hui. On reconnait, dans cette distribution, la présence d'une limite

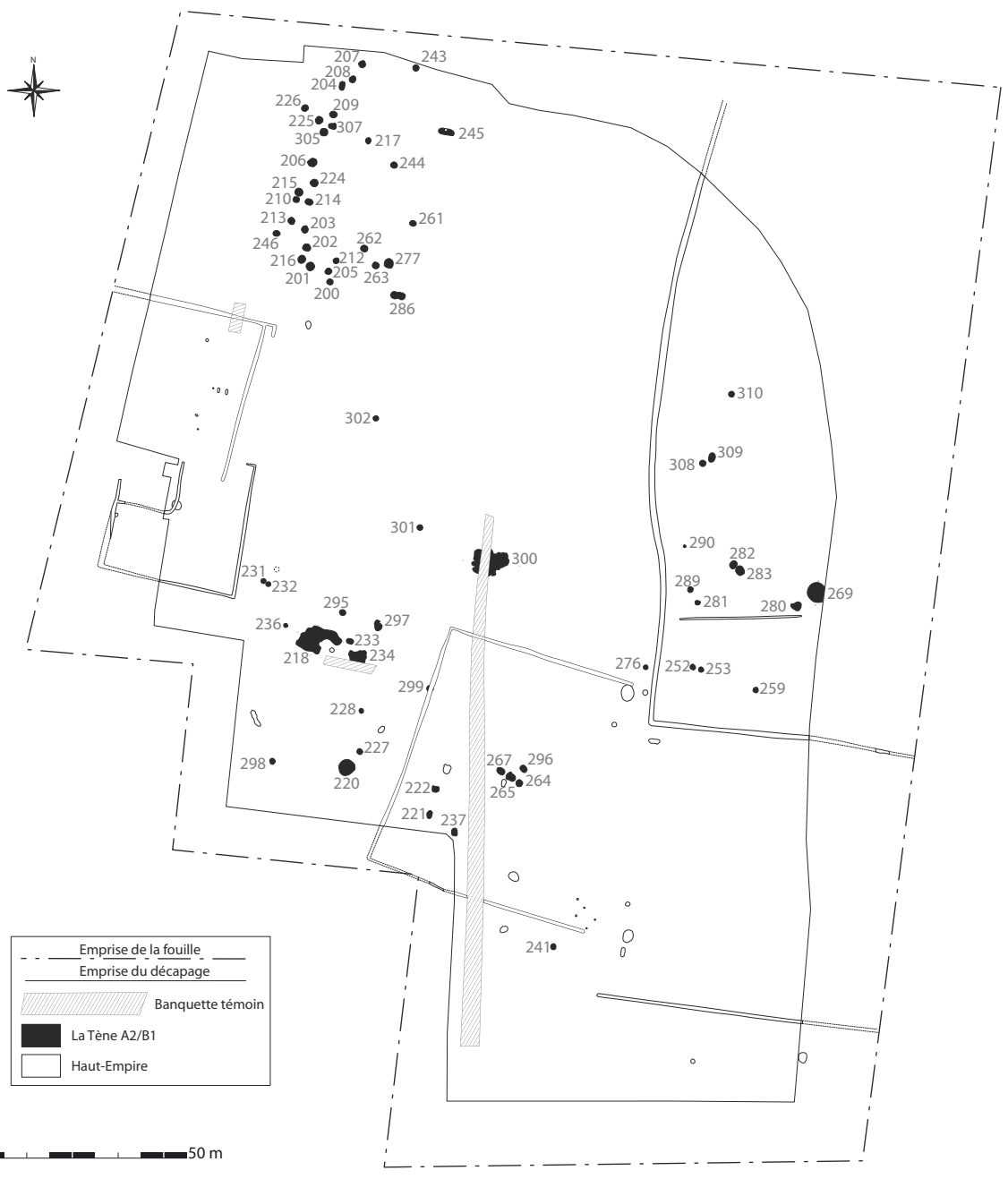

Figure 2 : Plan général de la fouille, hors parcellaire moderne. Topographie D. Fillon; DAO J.-M. Bryand.

Figure 2: General plan of the excavation except modern land registry. 


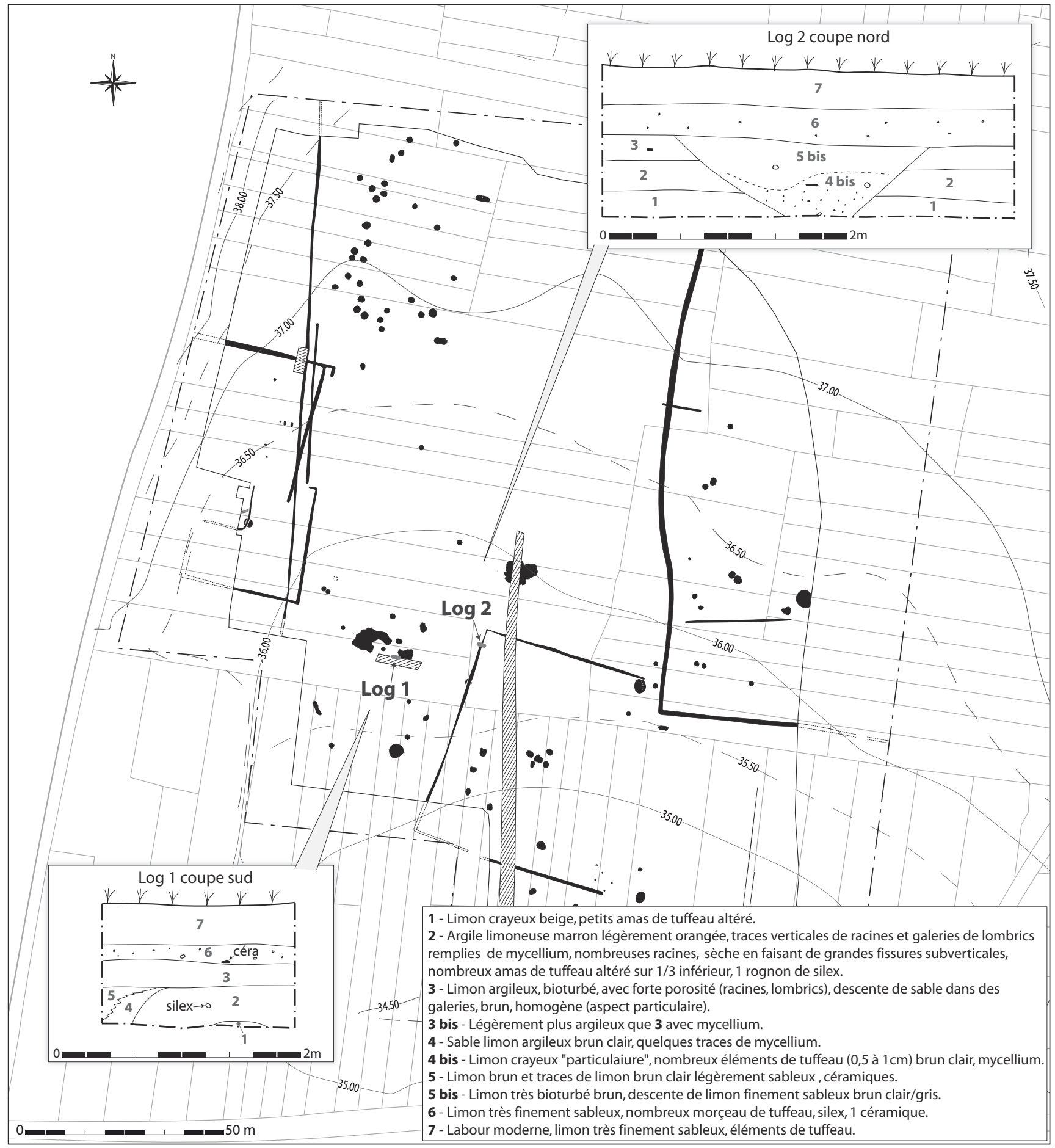

Figure 3 : Superposition des vestiges découverts en fouille sur le cadastre de 1810, localisation des logs. Topographie D. Fillon, DAO R. Arthuis, J.-M. Bryand.

Figure 3: Superimposing of remains discovered during excavation on the land registry of 1810, location of logs.

parcellaire matérialisée par une haie dense, voire d'un talus, qui suit la rupture de pente et correspond à la limite entre les parcellaires laniérés est-ouest et nord-sud dessinés sur le plan cadastral du $\mathrm{XIX}^{\mathrm{e}}$ siècle. Cette limite, par effet rideau, a stoppé ou diminué le colluvionnement provenant du nord de la parcelle. La colluvion de nature graveleuse (morceaux de craie), initiée par des techniques de labour mécanisées et alimentée par les éléments crayeux provenant du substratum 
tronqué au nord s'est accumulée le long de la haie. Elle a fossilisé le sol anthropisé qui tronque les structures de l'Âge du Fer et dans lequel est creusé le réseau fossoyé d'époque romaine (niveau 3, $\operatorname{logs} 1$ et 2 : fig. 3).

Par la suite, la disparition de la haie de partition entre les parcellaires laniérés nord et sud a permis une redistribution relative des sédiments accumulée au niveau de la haie vers le sud, dans le sens de la pente. Ceci explique l'épaisseur importante de sédiments dans la zone 35,5/36 m NGF et la conservation à cet endroit d'un sol ancien. Ailleurs, les sols sont tronqués.

Le substrat calcaire apparaît directement sous la terre végétale ou sous une couche d'argile d'altération du substrat pouvant atteindre plus d'un mètre d'épaisseur.

\section{L'occupation de La TÈne anCIENNE}

\section{Les structures}

La Tène ancienne est représentée par 69 fosses (fig. 2). Une grande partie correspond à des structures de stockage souterrain de type silo. Elles ne sont associées à aucun bâtiment. Ce phénomène n'est pas unique et l'on conclut souvent que ces batteries sont situées en périphérie du véritable habitat. Pour les Rogelins, les quantités de rejets domestiques dans les fosses ne permettent probablement pas de soutenir entièrement l'hypothèse. On ne peut totalement exclure la possibilité de l'évacuation des détritus loin des maisons, mais il semble plus raisonnable d'évoquer la disparition physique des unités d'habitation en raison de l'érosion des couches argileuses surmontant le calcaire. Le seul bâtiment conservé est d'époque romaine, les trous de poteau ne subsistent que sur une profondeur de quelques centimètres.

\section{Les structures de stockage souterrain}

Ce sont des fosses de plan circulaire ou subcirculaire, de profil tronconique. Des comparaisons archéologiques et ethnographiques permettent de les interpréter comme des silos (Gransar, 2000). D'autres fosses sont de forme cylindrique. Si elles sont bouchées hermétiquement, elles peuvent aussi correspondre à des silos. L'interprétation comme structure de stockage repose surtout sur la présence de ces fosses à proximité de silos bien identifiés.

- Les silos tronconiques fermés (fig. 4)

Ce sont les structures les plus nombreuses, avec 31 exemplaires. Elles ont un volume moyen de $2,6 \mathrm{~m}^{3}$, l'une atteint $5,77 \mathrm{~m}^{3}$. La profondeur moyenne est de 1,21 m.

Parfois, des effondrements ont modifié le profil d'origine (F. 243 : fig. 4). On peut penser que certains silos ont été comblés rapidement, les remplissages étant homogènes (F. 244 : fig. 4). Une petite couche argileuse horizontale à la base des fosses (F. 215 : fig. 4) peut témoigner de leur phase initiale d'utilisation. En effet, les parois des silos peuvent être enduites d'une couche d'argile pour augmenter leur étanchéité (Malrain et al., 2002). Par la suite, les comblements se font de façon subhorizontale (F. 215 : fig. 4) et/ou par apports latéraux (F. 201 : fig. 4). Une partie de ces remplissages s'est mise en place naturellement, mais ces structures ont également connu une dernière utilisation comme dépotoir.

- Les fosses tronconiques ouvertes (fig. 4)

Ces fosses présentent un profil légèrement évasé. Elles ont des dimensions comparables à celles des silos tronconiques fermés avec un volume moyen de $2,76 \mathrm{~m}^{3}$ pour une profondeur de $1,22 \mathrm{~m}$.

Pour F. 234 (fig. 4), on dispose d'une séquence stratigraphique du sommet de la terre végétale jusqu'au fond de la structure. Elle a été creusée (interface 2). Après comblement, l'ensemble a été recreusé une première fois (interface 1), puis partiellement une seconde fois (interface 3), ce qui n'est pas visible sur la coupe, car la fouille a été réalisée en palier.

- Étude micromorphologique de la fosse 234

Des prélèvements ont été réalisés en bordure de la fosse F. 234, au contact du substrat calcaire pour préciser le caractère en place ou remanié des couches. La séquence de dépôts supérieurs (niveaux IV à la base, V, VI et VII au sommet) a été étudiée pour identifier la présence de surfaces d'occupation (fig. 5).

L'Us 6 se distingue des Us 5 et 1 par sa composante plus carbonatée, dans la masse fine et dans le squelette minéral avec plus de grains carbonatés que de sables siliceux. Elle provient d'un niveau de sol sans doute proche du substrat calcaire mais renferme aussi des agrégats de texture différente, limono-argileux à argilo-limoneux brun-jaune ou bruns et agrégats sablo-limoneux carbonatés qui témoignent d'un mélange de sédiment. De rares charbons et un fragment de graine carbonisée (fig. 6) sont aussi incorporés dans ce sédiment remanié. La structuration en plages grumeleuses à micro-agrégées, des agrégats tubulaires et des chenaux témoignent de l'impact de la faune du sol. Des hyporevêtements carbonatés, des pseudomorphoses calcitiques relèvent probablement de l'activité racinaire (Becze-Deak et al., 1997).

L'Us 5 est composée d'un mélange de sables carbonatés et siliceux avec des plages de grains plus carbonatés et davantage de plages argilo-limoneuses à limono-argileuses brun-jaune non carbonatées. Elle évoque différentes sources d'apports, notamment celui d'un niveau de sol similaire à la couche 


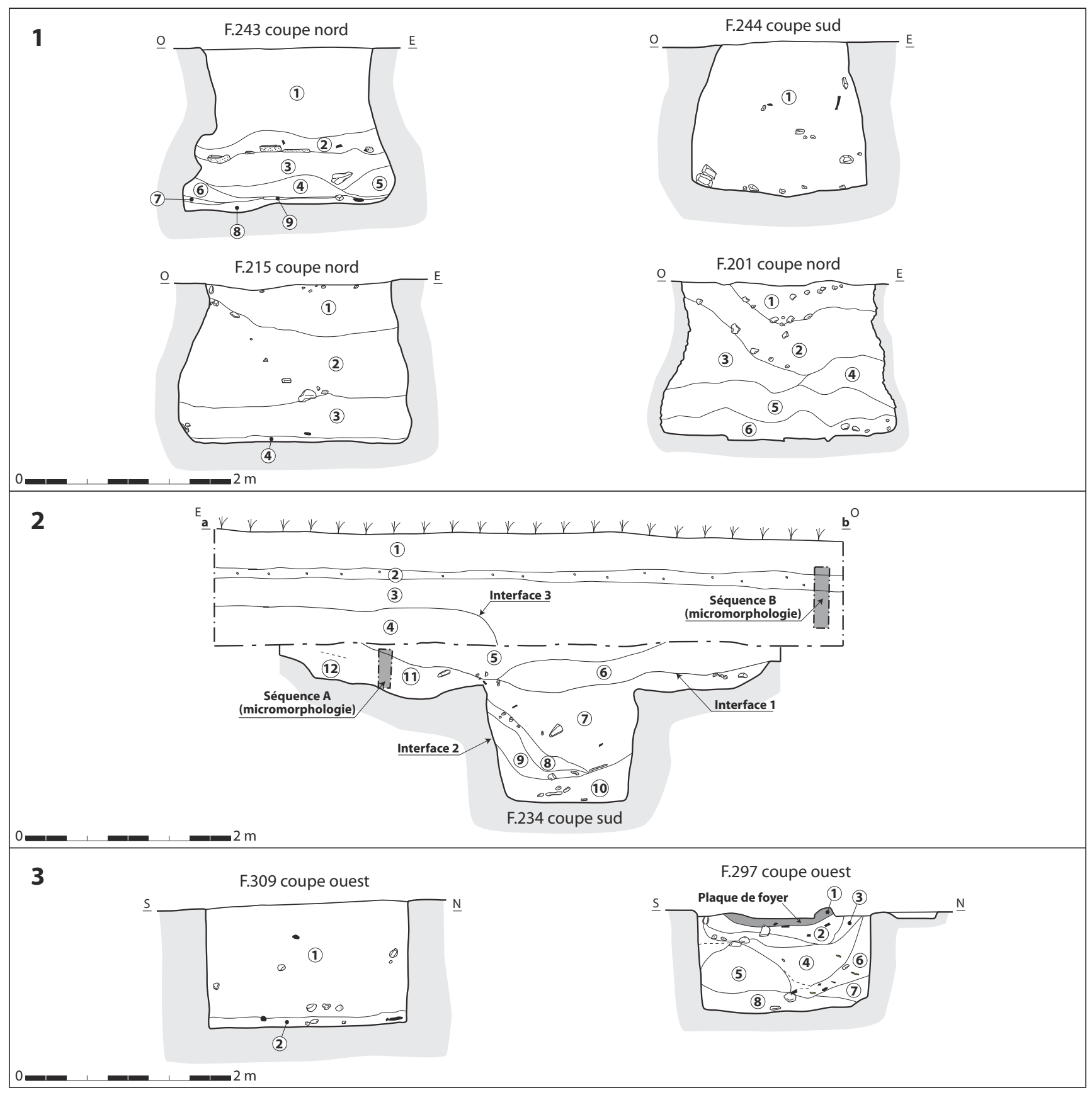

Figure 4 : Les silos (DAO J.-M. Bryand).

Figure 4: The storage pits.

IV de la séquence supérieure. Des charbons et de rares fragments d'os sont relevés. De rares revêtements limoneux ou limoneux finement sableux déposés sur les vides résultent de percolations. Enfin, des chenaux attestent d'une activité biologique qui affecte également la couche inférieure 6. Cette couche hétérogène peut résulter d'une redistribution d'origine anthropique ou d'épisodes d'apports (érosion?) et d'une exposition temporaire aux agents naturels.
Dans l'Us 1, la matrice et l'impact des agents naturels sont similaires à ceux de l'Us 5 . Les constituants anthropiques sont sensiblement plus diversifiés (charbons, fragments d'os et de céramique, agrégats en terre crue résiduels), intégrés dans un mélange de matériaux sédimentaires. 


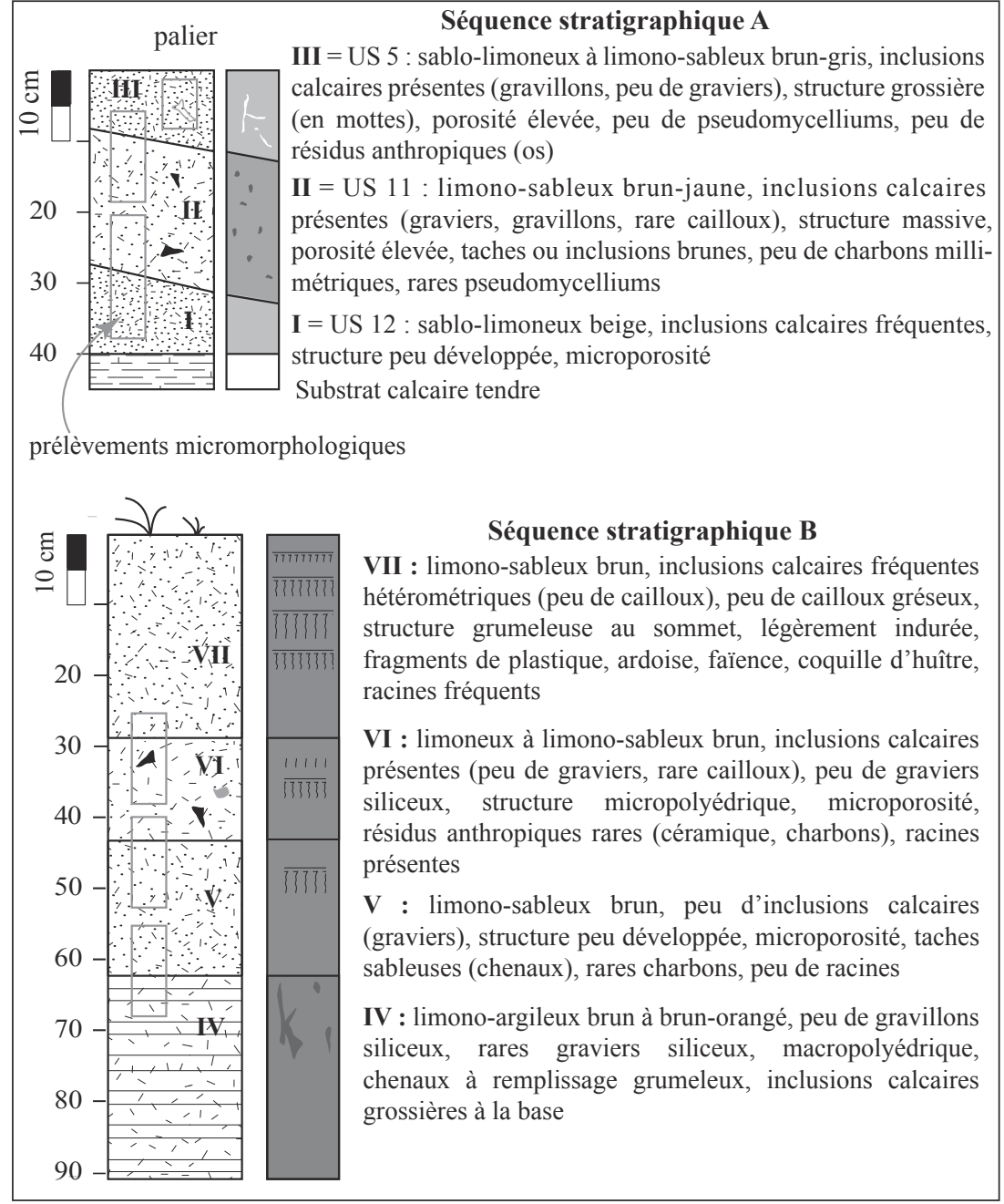

Figure 5 : Fosse 234, caractères macroscopiques des couches étudiés (DAO C. Vissac). Figure 5: Pit 234, description of studied deposits in the field.

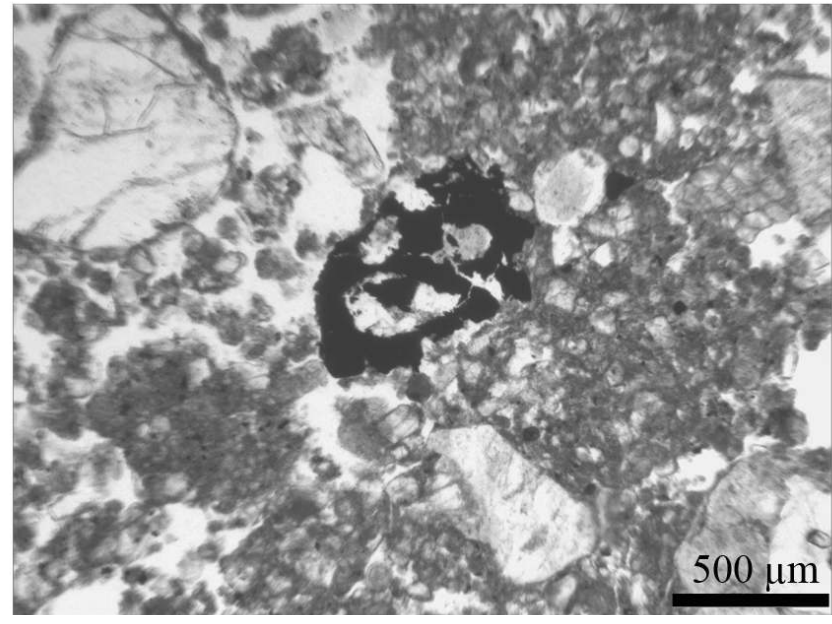

Figure 6 : Fosse 234, fragment de graine carbonisée dans la couche 1, observation au microscope polarisant, lumière polarisée non analysée. Cliché C. Vissac.

Figure 6: Pit 234, fragment of carbonised seed in deposit 1, photograph of thin section in plane polarised light.
- La séquence de dépôts supérieurs

La couche IV est caractérisée par une matrice argileuse non carbonatée avec présence de sables siliceux et de résidus de dissolution carbonatés. La formation de revêtements argileux jaunes à brun-jaune progressivement intégrés à la matrice a été favorisée par la décarbonatation du sédiment. D'autres revêtements argileux plus poussiéreux bruns indiquent par contre des percolations sur un sol déstabilisé (Courty et Fédoroff, 2002).

La transition avec la couche $\mathrm{V}$, hormis des échanges verticaux liés à l'activité biologique, est assez nette en raison d'une différence de composition. Celle-ci montre davantage d'éléments carbonatés en particulier sous forme de sables ou de fragments rocheux non altérés. Les charbons sont également plus grossiers. La structure du sédiment est marquée par l'impact de l'activité biologique comme en témoignent des plages grumeleuses et des biosphéroïdes produits par la faune du sol (Becze-Deak et al., 1997). Des imprégnations ferrugineuses, de concentrations sableuses ou de masse fine 
évoquent toutefois des redistributions en contexte humide saturé, internes au sédiment ou par des apports hydriques superficiels.

La couche VI est similaire mais renferme quelques résidus d'activités diversifiés (céramique, os, charbons, granules phosphatées de type déjection?) notamment concentrés vers la base avec de rares lits discontinus de grains sableux et carbonatés qui suggèrent la présence d'anciennes surfaces peutêtre fréquentées comme le montrent des assemblages plus denses subhorizontaux (fig. 7) une réduction de la porosité ou des microfissures (Gé et al., 1994).

La couche VII correspondant à l'horizon de surface actuel montre sensiblement plus d'éléments carbonatés (fragments rocheux) avec des degrés d'altération variables ainsi que des constituants hétérogènes visibles sur le terrain (ardoise, faïence, résidus plastique, coquilles d'huître). Elle provient notamment d'apports anthropiques (rejets). Des fissures subhorizontales sont attribuables à l'impact du travail du sol (semelle de labours).

\section{Conclusion}

Aux abords de la fosse, l'étude micromorphologique met en évidence des couches remaniées correspondant probablement aux phases de comblement. La composition des couches supérieures correspondant notamment au niveau de creusement relève du développement d'un sol d'apport colluvial en lien avec l'occupation du site (mise en culture, redistribution de matériaux, rejets...).

-Les fosses cylindriques (fig. 4)

Ce sont des petites structures avec un volume moyen de $1,41 \mathrm{~m}^{3}$ pour une profondeur de $0,88 \mathrm{~m}$.

Les comblements sont assez simples, il s'agit le plus souvent de remplissages homogènes (F. 309 : fig. 4). La fosse 297 (fig. 4) a connu une utilisation secondaire. C'est probablement à la suite d'un effondrement d'une paroi (Us 5) que la fonction première de la structure a été abandonnée. Un foyer plusieurs fois activé a induré la partie supérieure du comblement sur lequel il était installé.

\section{Conclusion}

L'ensemble de ces fosses témoigne d'une capacité de stockage importante. Le volume cumulé actuel des silos est de $99,8 \mathrm{~m}^{3}$, si l'on ajoute les fosses tronconiques ouvertes et les cylindriques, on atteint $115,1 \mathrm{~m}^{3}$. Ces chiffres sont obtenus pour des structures érodées. La partie détruite correspond en grande partie à la cheminée d'accès. Au moment de la fermeture du silo, ce conduit était comblé de paille grossière, puis de paille fine, de façon à garantir une atmosphère confinée, elle ne contenait pas de denrées (Malrain et al., 2002). Il est donc assez probable que le volume manquant correspondant

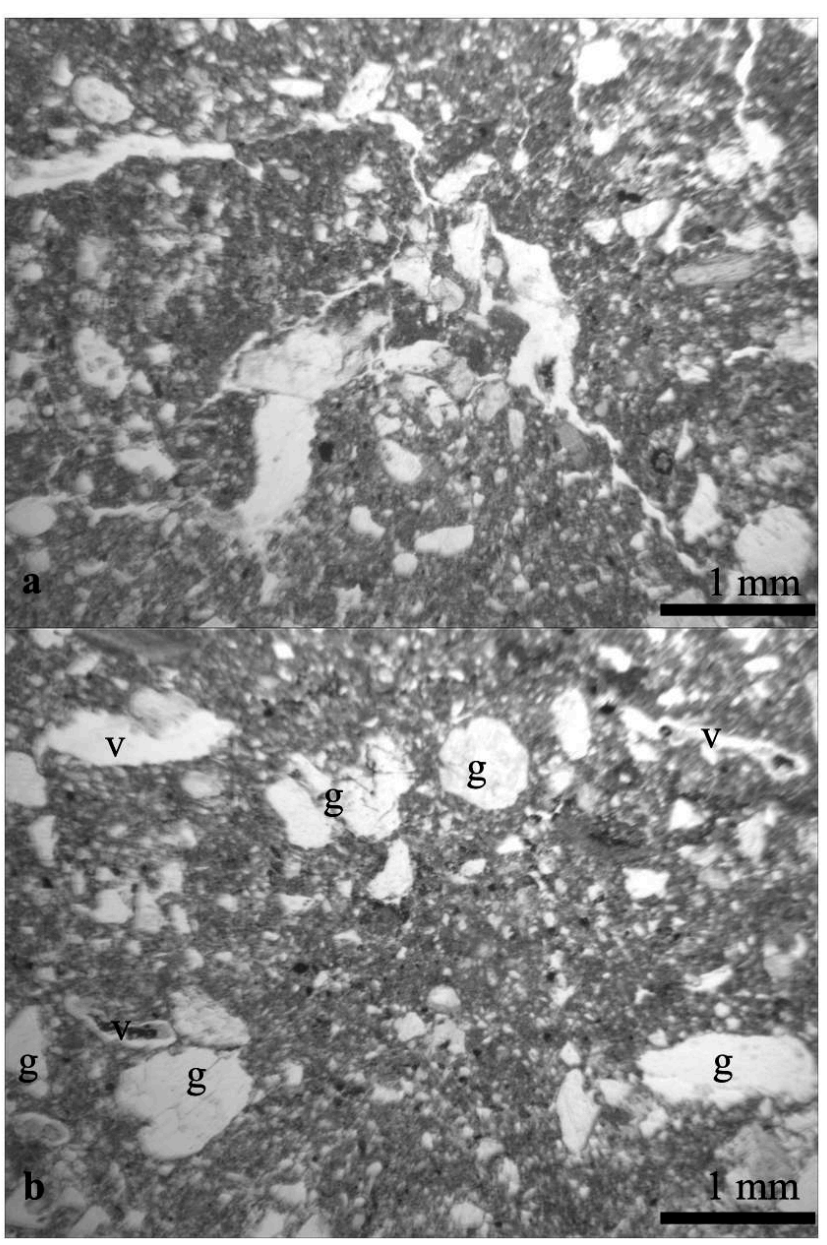

Figure 7 : Fosse 234, a : couche VI, organisation d'origine biologique. $\mathrm{b}$ : couche VI, lits de grains sableux avec assemblages denses subhorizontaux, $\mathrm{g}$ = grains minéraux, $\mathrm{v}=$ vide aplati, observations au microscope polarisant, lumière polarisée non analysée. Cliché C. Vissac.

Figure 7 : Pit 234, a: deposit VI, excremental fabric, b: deposit VI, layering of discontinous mineral components and dense unit, $g=$ mineral component, $v=$ horizontal void, photograph of thin section in plane polarised light.

majoritairement à la cheminée influe assez peu sur le calcul du volume initial de stockage utile. Il est probablement assez proche du volume actuel. Si l'on prend l'exemple du blé $\left(800 \mathrm{~kg} / \mathrm{m}^{3}\right)$, en fonction d'une base de calcul établie à partir d'un taux d'érosion compris entre $10 \%$ et $50 \%$ des fosses et de la prise en compte des seuls silos ou de toutes les structures ayant potentiellement la même fonction, on obtient une capacité de stockage comprise entre 79,8 et 138,1 tonnes. Le chiffre est maximal, dans la mesure où il concerne l'ensemble des structures si elles avaient servi pendant toute la durée de l'occupation.

Pour restituer les réserves alimentaires disponibles aux Rogelins, on prendra l'exemple du pain. On part du pos- 
tulat qu'il est réalisé avec une farine intégrale (avec la balle ou son : type 150 : norme NF V03-720/ISO 2171) plus riche en fibres et en vitamines que le pain actuel, majoritairement élaboré dans des farines pauvres (type 55). On compte un kilo de pain par jour et par personne sans tenir compte de la taille ou du sexe des individus. Pour un kilo de pain à partir de ce type de farine, il faut 750 grammes de blé. Avec un taux d'extraction de 90 à $98 \%$, cela correspond à 705 grammes de farine. Des valeurs moyennes, entre 30 et $60 \%$ des structures utilisées simultanément et une érosion de 10 à $20 \%$ permettent de calculer une nourriture suffisante pour 103 à 258 personnes pendant un an.

\section{La céramique}

La céramique de Chacé est représentée par 6138 NR pour 731 NMI, 270 formes de vases ou NTI ont été classées.

\section{Méthode de classement}

Les céramiques sont différenciées selon deux critères : hauteur et ouverture, selon un protocole couramment utilisé.

- Forme basse : hauteur du vase (sans un éventuel pied) $\leq$ au diamètre de l'ouverture.

- Forme haute : hauteur du vase (sans un éventuel pied) $>$ au diamètre de l'ouverture.

- Forme ouverte : diamètre de l'ouverture $\geq$ au diamètre maximum.

- Forme fermée : diamètre de l'ouverture < au diamètre maximum.

Ces critères se combinent pour former des groupes :

- Groupe A : formes basses ouvertes;

- Groupe B : formes basses fermées;

- Groupe C : formes hautes ouvertes;

- Groupe D : formes hautes fermées.

À l'intérieur de ces groupes, les types sont définis selon la morphologie de leur profil (panse, épaule, col). Les variantes sont établies par des critères secondaires propres à chaque type, ils sont multipliés jusqu'à l'individualisation du vase.

\section{Typologie (fg. 8)}

- Groupe A : formes basses ouvertes

- Type A1 : profil tronconique - La forme générale est tronconique, elle se caractérise par l'absence de col. Le critère discriminant les variantes repose sur la forme de la lèvre.

- A10 : lèvre simple.

- A11 : lèvre relevée.

- A12 : lèvre ourlée.
La variante A12 est une jatte ou coupe à bord festonné, ourlé, à indentation ou une coupe parasol selon les auteurs. En premier lieu, c'est M.-E. Mariën (1961) qui décrit cette forme et suggère une utilisation comme brasero ou luminaire. Pour G. Leman-Delerive (1973), il s'agit d'un ustensile dont les bords festonnés servent à évacuer le lait pour fabriquer la crème. B. Lambot réalise un nouvel inventaire (Lambot, 1988) et propose à nouveau une interprétation comme luminaire. Les bords festonnés pourraient être propices à recevoir des mèches imbibées dans de la graisse ou de l'huile. La faible contenance du récipient s'accorde avec cette interprétation. Leur présence dans des habitats et surtout dans des grottes est un argument parfois avancé pour lui attribuer cette fonction. Cette idée est reprise à propos d'une synthèse pour le nord de la France (Hurtrelle et al., 1990) et de nouveau argumentée par la découverte d'un exemplaire dans un petit atelier couvert. P.-Y. Milcent (2006) étudie ce type dans le cadre de sa définition culturelle du premier Age du Fer médio-atlantique. Il note, comme M.-E. Mariën, qu'il pourrait aussi s'agir de vases de cuisson sur braise et conclut qu'il s'agit au moins d'un ustensile domestique original. À Mervent (Nillesse et al., 2014) il est associé à une céramique basse et plate interprétée, elle aussi, comme un luminaire.

- Type A2 : profil bipartite - Ce type est caractérisé par une panse tronconique qui s'articule sur un col cylindrique. - A20 : une seule variante.

- Type A3 : profil sinueux - Les vases de ce type ont une panse s'articulant sur une carène ou une épaule se terminant par un col ouvert. La forme de la panse et du col déterminent des variantes.

- A30 : panse ronde, col tronconique ouvert.

- A31 : panse tronconique, col concave.

- A32 : panse tronconique (?), col pseudo-cylindrique.

- A33 : panse tronconique, col tronconique ouvert.

- Groupe B : formes basses fermées

- Type B1 : profil bipartite, bord rentrant - Le type possède une panse tronconique qui se referme et se terminant par un bord rentrant, c'est la classique jatte à bord rentrant.

- B10 : une seule variante.

- Type B2 : profil bipartite, épaule rentrante - La panse tronconique ouverte s'articule sur une épaule tronconique fermée.

- B20 : une seule variante.

- Type B3 : profil sinueux, épaule non saillante - Le schéma de base de ce vase est tripartite (panse, épaule, col). Ces trois parties forment un segment continu et sinueux. L'épaule n'est pas saillante (elle ne dépasse pas l'aplomb de l'extrémité de la lèvre). 


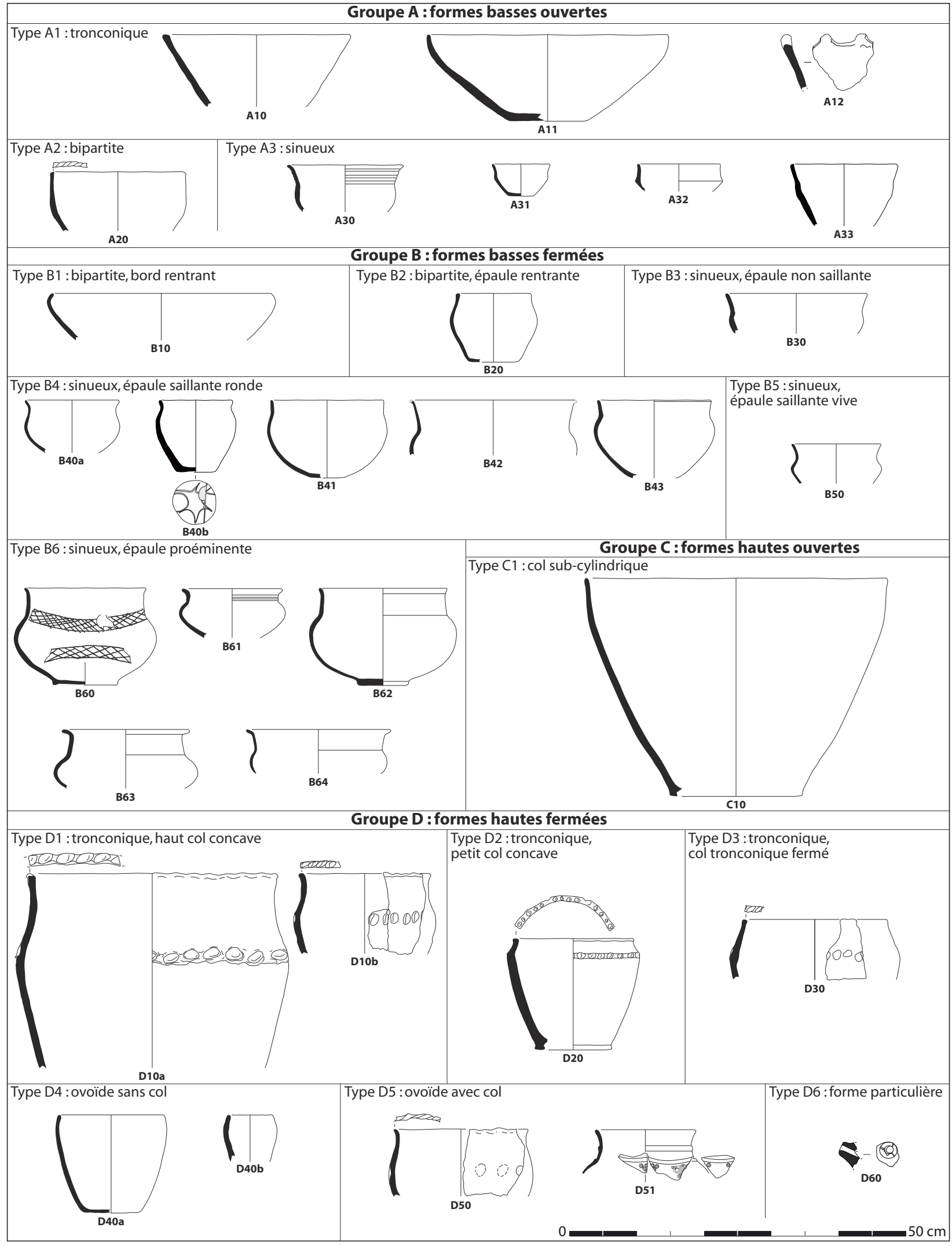

Figure 8 : Typologie de la céramique. DAO J.-M. Bryand.

Figure 8: Ceramic typology. 
- B30 : une seule variante.

- Type B4 : profil sinueux, épaule saillante ronde - Les vases de ce type ont un profil sinueux. L'épaule ronde est plutôt saillante (elle est à l'aplomb de l'extrémité de la lèvre, ou la dépasse légèrement). Les variantes sont établies par la forme du col. Les variantes sont distinguées par la forme du col. Haut col : hauteur du col $>15 \%$ de la hauteur du vase.

- B40a : col concave, modèle bas.

- B40b : col concave, modèle à la limite des vases hauts.

- B41 : col cylindrique.

$-\mathrm{B} 42$ : col tronconique ouvert haut.

- B43 : col tronconique ouvert bas.

- Type B5 : profil sinueux, épaule saillante vive - le profil est identique à celui de $\mathrm{B} 4$, mais l'épaule est carénée.

- B50 : une seule variante.

- Type B6 : profil sinueux, épaule proéminente - Le profil est encore sinueux, l'épaule est proéminente (elle dépasse l'aplomb de l'extrémité de la lèvre ou le point d'inflexion entre l'épaule et la lèvre, quand celle-ci est divergente). Les variantes se déclinent à partir de la forme du col et de la lèvre.

- B60 : col peu concave, lèvre simple.

- B61 : col concave, lèvre simple.

- B62 : col cylindrique, lèvre simple.

- B63 : col tronconique fermé, lèvre divergente.

- B64 : col tronconique ouvert, lèvre divergente.

Groupe $\mathrm{C}$ : formes hautes ouvertes

- Type C1 : col sub-cylindrique - La forme générale est tronconique ou légèrement galbée, elle s’articule sur un col ouvert.

- C10 : une seule variante.

- Groupe D : formes hautes fermées

- Type D1 : profil tronconique, haut col concave - Le type possède une panse tronconique ou peu galbée qui s'articule au niveau de l'épaule adoucie sur un haut col peu concave ou concave.

- D10 : une seule variante, deux modules sont présentés.

- D10a : grand module.

- D10b : petit module.

- Type D2 : profil tronconique, petit col concave - La forme générale du vase est identique à celle de $\mathrm{D} 1$, mais le col est court. Petit col : hauteur du col $<20 \%$ de la hauteur du vase.

- D20 : une seule variante.

- Type D3 : profil tronconique, col tronconique fermé La forme générale est encore celle de D1, mais le col est ici tronconique fermé.

- D30 : une seule variante.
Ces trois types (D1, D2, D3) ont souvent été désignés comme des situles, imitations des prototypes métalliques pouvant être destinés au service du vin. Comme P.-Y Milcent (2006) le souligne, c'est un abus de langage, la forme s'inspire de modèles locaux enracinés depuis le Bronze final.

- Type D4 : profil ovoïde, sans col - Les vases de cette série possèdent une panse ovoïde dont le diamètre maximum (épaule) est situé au-dessus de la moitié de la hauteur du vase. La partie supérieure du vase est rentrante, mais ne constitue pas un col.

- D40 : une seule variante, deux modules sont présentés.

- D40a : grand module.

- D40b : petit module.

- Type D5 : profil ovoïde, avec col - Ces vases ont une panse ovoïde dont le diamètre maximum (épaule) est situé vers ou sous la moitié de la hauteur du vase. Le rétrécissement de la panse vers le haut du vase constitue le point d'inflexion avec le col. Les variantes sont déterminées par la forme du col.

- D50 : col peu concave, peu individualisé.

- D51 : col concave, bien individualisé.

- Type D6 : forme particulière - Le goulot appartient à une série rare, il est attesté sur un vase de la tombe d'Andonne à Villejoubert en Charente (Debord et al., 2000) bien datée du Hallstatt C2 par une épée à languette tripartite et une hache en fer. Un peu plus tard, on retrouve le goulot ou bec verseur sur un vase de la tombe 87 HIJ 19 d'Antran, datée du Hallstatt D1 (Pautreau, 1991) puis dans la Vienne au Camp Allaric (Maitay, 2008).

- D60 : une seule variante.

\section{Comparaisons}

- Typologie

Les sites de comparaison pour les Rogelins sont particulièrement rares. En effet, si la fin du premier Âge du Fer et le début du second sont assez bien documentés (Hallstatt D3/ La Tène A1), il n'est pas de même pour une grande partie de La Tène ancienne (La Tène A2/La Tène B2). Trois séries seulement sont suffisamment importantes pour offrir des parallèles valables (fig. 9 à 11) : la Croix Boizard à Brion (Barbier, 1995), le Petit Cabaret à Cizay-la-Madeleine (Levillayer et al., 2013) dans le Maine-et-Loire et la Renaîtrie à Chatellerault dans la Vienne (Poirier et al., 2005).

Les types A11 (forme ouverte) et B10 (forme fermée) procèdent du même schéma, seule la forme de la lèvre les différencie. A11 est une forme très fréquente, elle se développe pendant les deux Ages du Fer. La variante fermée, la jatte à bord rentrant, apparaît dès le Hallstatt D3 à Saint-Hilaire/Saint-Florent dans le Maine-et-Loire (Bouvet, 1987), mais elle se développe surtout à partir de La Tène ancienne comme à la Croix Boizard, au 


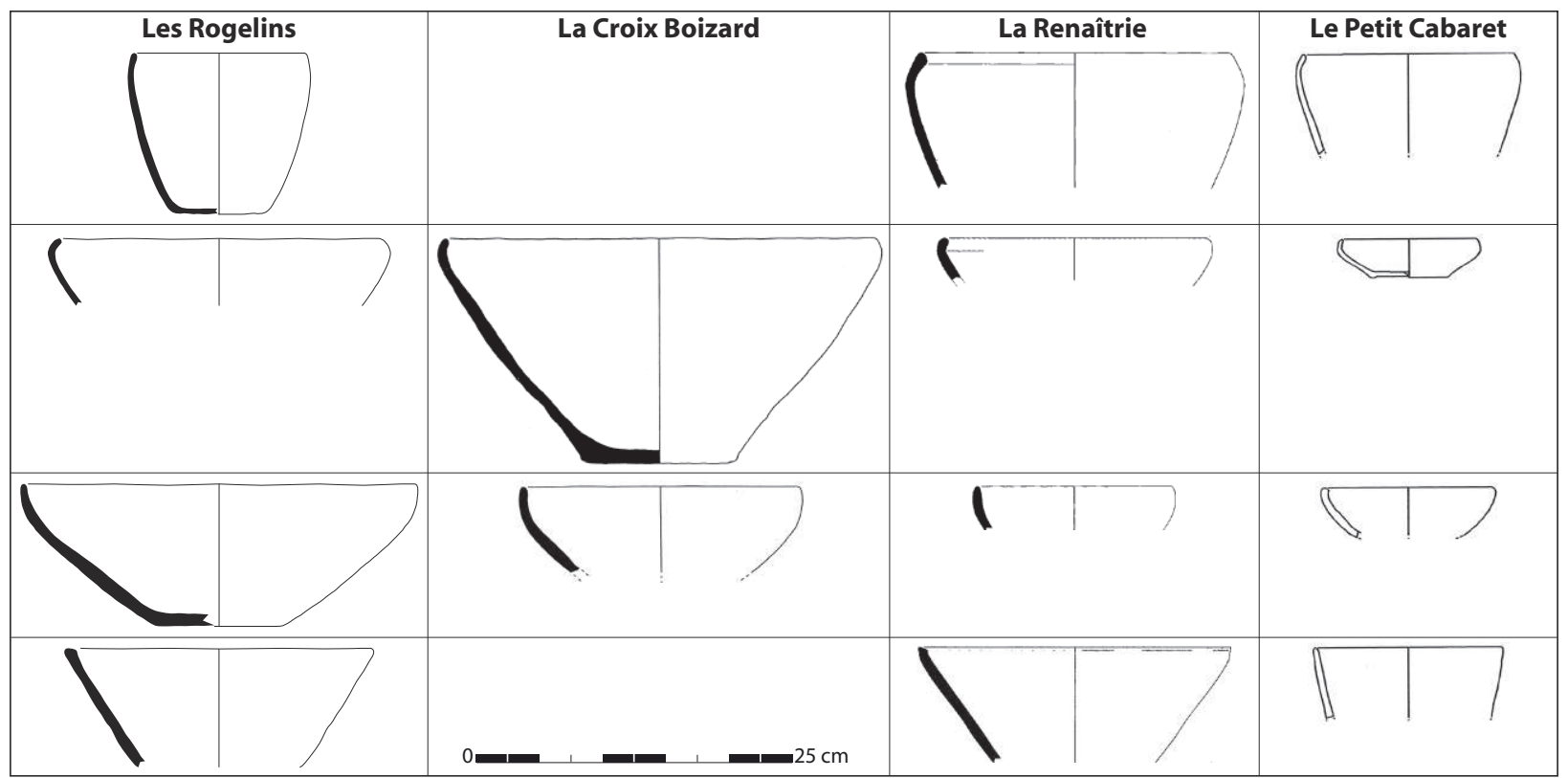

Figure 9 : Comparaisons entre les céramiques des Rogelins, de la Croix Boizard (d'après Barbier, 1995), de la Renaîtrie (d'après Poirier et al., 2005) et du Petit Cabaret (d'après Levillayer et al., 2013).

Figure 9: Comparisons between ceramic of Rogelins, la Croix Boizard (after Barbier, 1995), of la Renaîtrie (after Poirier et al., 2005) and of Petit Cabaret (after Levillayer et al., 2013).

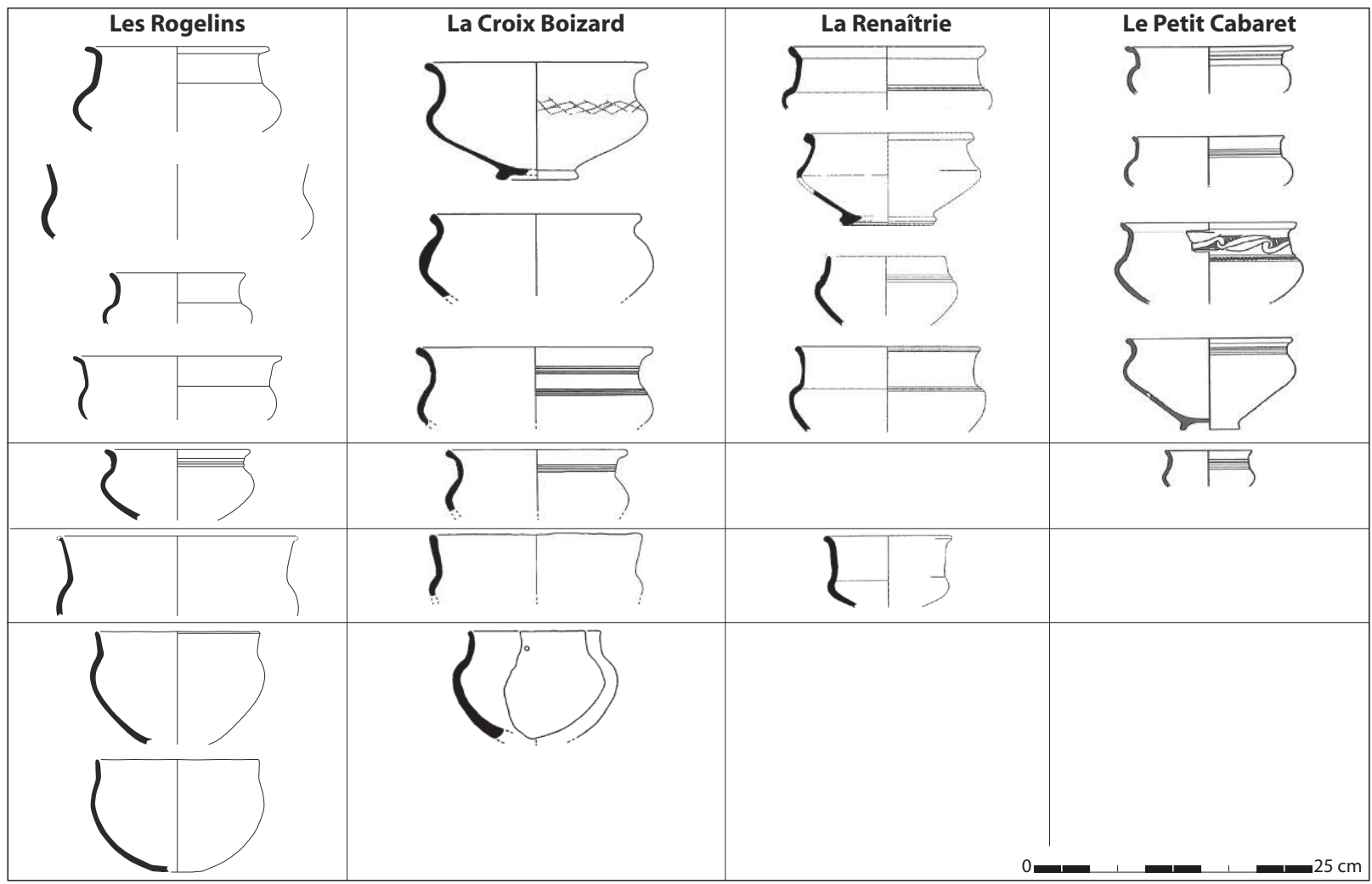

Figure 10 : comparaisons entre les céramiques des Rogelins, de la Croix Boizard (d'après Barbier 1995), de la Renaîtrie (d'après Poirier et al., 2005) et du Petit Cabaret (d'après Levillayer et al., 2013).

Figure 10: Comparisons between ceramic of Rogelins, la Croix Boizard (after Barbier, 1995), of la Renaitrie (Poirier et al., 2005) and of Petit Cabaret (after Levillayer et al., 2013). 


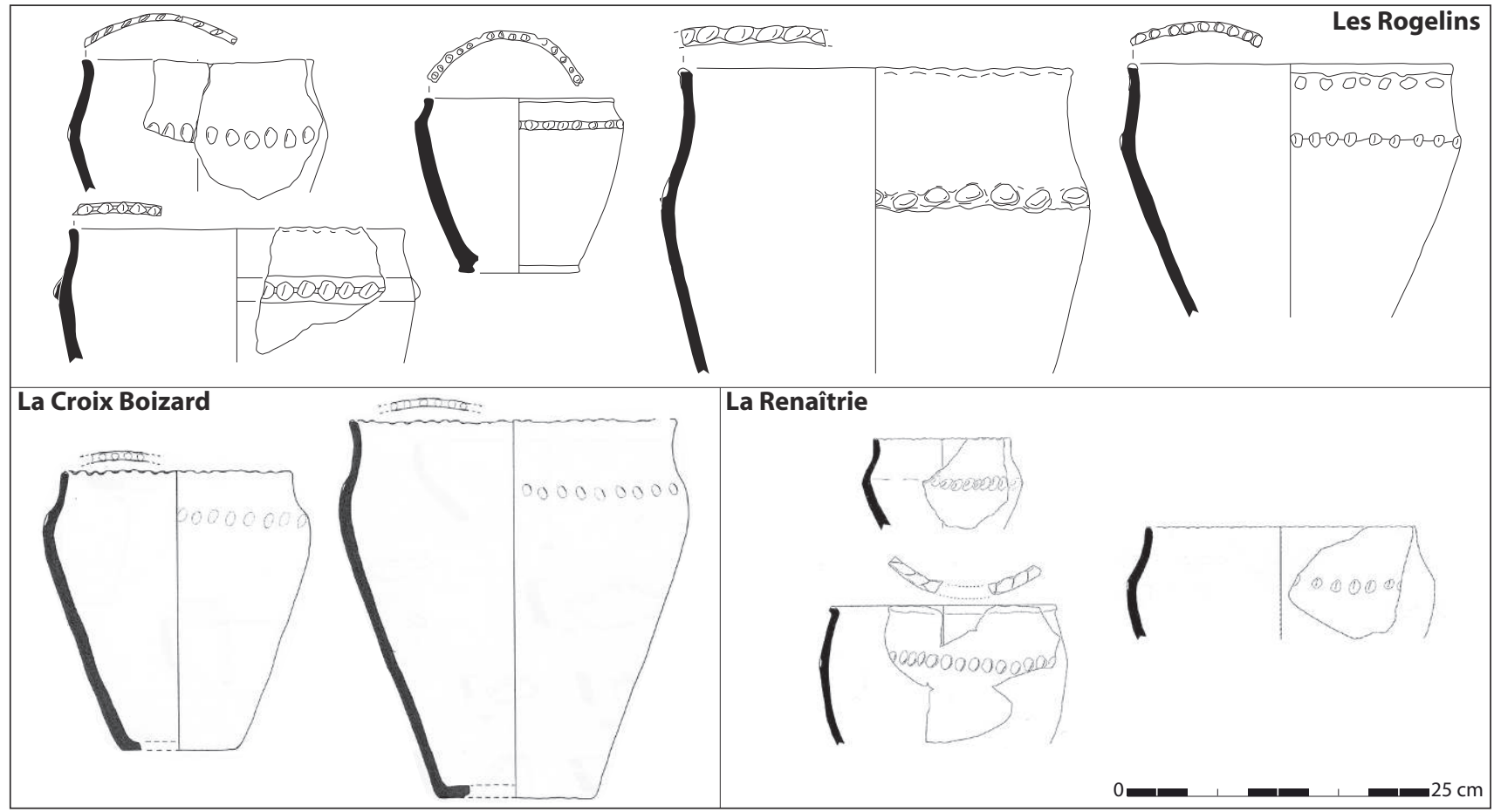

Figure 11 : Comparaisons entre les céramiques des Rogelins, de la Croix Boizard (d'après Barbier 1995) et de la Renaîtrie (d'après Poirier et al., 2005).

Figure 11: Comparisons between ceramic of Rogelins, la Croix Boizard (after Barbier, 1995) and of la Renaîtrie (after Poirier et al., 2005).

Petit Cabaret et à la Renaîtrie. Jusqu'à la transition La Tène C2/ D1a, elle est quantitativement inférieure à l'écuelle à profil en S. À partir de La Tène D, la jatte à bord rentrant ne subsistera qu'au sud de la Loire où sa proportion par rapport aux écuelles à profil en $S$ est un indicateur chronologique et culturel (Buchsenschutz et al., 1993; Nillesse, 2012). D40 est la variante haute de $\mathrm{B} 10$, on la trouve à la Renaîtrie.

Pour les formes basses fermées (groupe B), les types B61, B63 et B64 présentent des caractères particuliers comme des lèvres divergentes, des hauts cols ou des panses proéminentes comme on en trouve sur les trois sites de référence. B64 est également connu dans le Maine-et-Loire à Chanzeaux, la Morinière (Viau et al., 1999). Une variante de B61 trouve une comparaison assez juste à la Croix Boizard et au Petit Cabaret. Le type B42 possède un col tronconique ouvert qui s'articule sur une panse bien développée, la lèvre est simple. Cette morphologie apparaît à la Croix Boizard. À la Renaitrie, le vase est plus éloigné de ce modèle et il se rapproche plus d'une évolution du mal nommé « gobelet jogassien ». Enfin, les types B41 et B43 s'apparentent à une forme de la Croix Boizard.

La seule forme haute ouverte (C10) n'appelle pas de remarques particulières.

Les formes hautes fermées D10, D20 et D30 sont élaborées sur un même modèle : panse tronconique haute s'articu- lant sur une épaule plus ou moins marquée qui ferme le vase. Ils comportent des décors digités ou des cordons rapportés. Ces vases sont réalisés dans des argiles grossières et il n'y a pas eu de soucis de reproduire strictement la même forme.

\section{- Composition du corpus}

Les formes basses ouvertes (groupe A) sont les plus fréquentes avec $46 \%$ du NTI, elles sont suivies des formes basses fermées (groupe B, $31 \%$ ) et des formes hautes fermées (groupe D, $23 \%$ ). Les formes basses ouvertes (groupe C) sont en quantité négligeable avec un seul individu. Le mobilier des Rogelins ayant été enregistré de la même façon que celui du site de Mervent, attribué au Hallstatt D3 (Nillesse et al., 2014), des comparaisons quantitatives sont possibles (fig. 12).

Les proportions des groupes A et B pour les deux sites sont inversées. À Mervent les formes basses fermées (groupe B) sont largement majoritaires avec $57 \%$ de l'effectif (31\% aux Rogelins). Au contraire, à Mervent, les formes basses ouvertes (groupe A) ne représentent que $21 \%$ du total contre $46 \%$ aux Rogelins. Les proportions des vases hauts ouverts sont proches, mais elles ne reposent pour chaque site que sur un individu. Enfin, les taux des formes hautes fermées sont équivalents entre les deux corpus. 


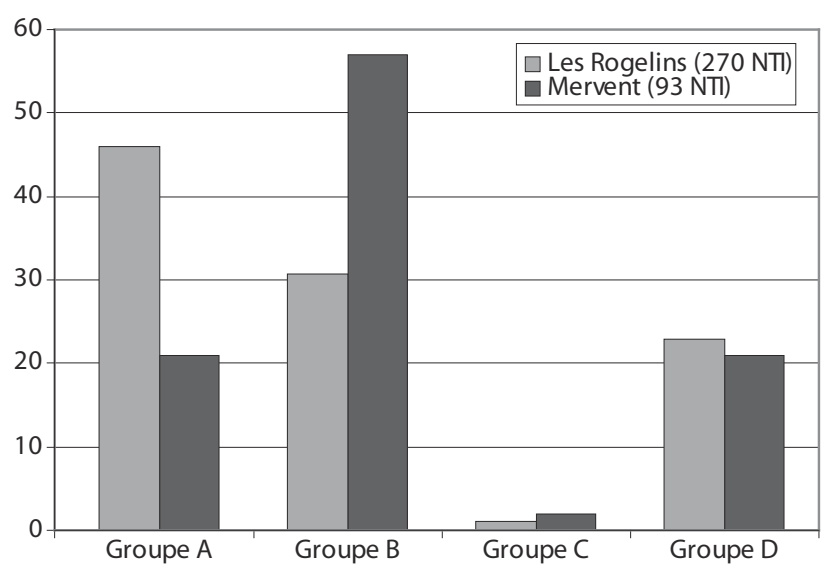

Figure 12 : Proportions des groupes typologiques aux Rogelins et à Mervent. DAO J.-M. Bryand.

Figure 12: Proportions of the typological groups in Rogelins and in Mervent.

On peut proposer une interprétation de ces résultats en termes d'évolution technique. À Mervent, avant le milieu du $\mathrm{v}^{\mathrm{e}}$ s. av. J.-C., on trouve plus de formes complexes (groupe B) que de formes simples (groupe A). Les premières sont basées sur une construction bi ou tripartite. Les éléments constitutifs du vase, des plaques, étant assemblées pour obtenir le résultat voulu. Un pied creux est parfois la troisième ou quatrième partie du récipient. L'investissement technique est plus important que pour réaliser des formes tronconiques ou en partie hémisphériques dont les fonds sont toujours plats. Aux Rogelins, à la fin du v ve s. av. J.-C., le potier produit des formes plus simples et plus rapides à réaliser.

- Groupes et types de productions :

approche macroscopique

242 NTI ont fait l'objet d'une description de leur pâte et d'un enregistrement de leur mode de cuisson (Picon 1973).

Modes de cuisson - Pour les Rogelins, c'est le mode B (cuisson réductrice, post-cuisson réductrice) qui domine largement avec plus de $65 \%$ des vases, c'est exactement l'inverse à Mervent où le mode $\mathrm{A}$ (cuisson réductrice, postcuisson oxydante) concerne plus de $70 \%$ des vases (fig. 13).

À Mervent, la maîtrise d'une cuisson réductrice alliée à une post-cuisson oxydante a permis d'obtenir des variations chromatiques intéressantes. Des effets de glacis ou de transparence ont abouti à des tons très particuliers. L'enfumage après la post-cuisson oxydante a donné des aspects noirs avec un fond rouge sous-jacent. Ces réalisations ont nécessité une parfaite maîtrise technique de la cuisson, on peut même envisager des fours à chambres séparées. Le mode B est plus facile à mettre en œuvre, une cuisson en fosse que l'on couvre de terre en post-cuisson suffit. On peut également boucher le four, les effets obtenus sont alors plus ternes.

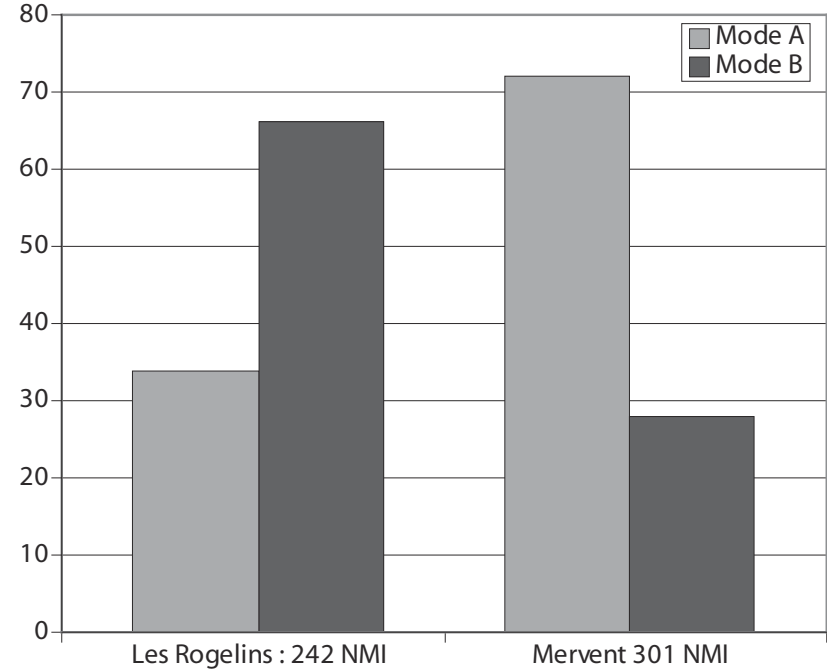

Figure 13 : Modes de cuisson des céramiques aux Rogelins et à Mervent. DAO J.-M. Bryand.

Figure 13: Modes of cooking of ceramic in Rogelins and in Mervent.

\section{- Classement des pâtes en groupes}

L'examen macroscopique du matériel a permis de distinguer trois grandes catégories en fonction de la taille des inclusions (argile fine, semi-fine, grossière). Les descriptions sont faites à l'œil nu et non sous binoculaire, et sans mesure des inclusions. Étant donné l'imprécision de la méthode, on ne cherchera à dégager que de grandes orientations. Lépaisseur du tesson (fin, épais) a été combinée aux trois grandes catégories, ce qui permet d'obtenir six groupes (fig. 14).

Ce sont les céramiques grossières (parois épaisses et parois fines cumulées) qui dominent avec $41 \%$ du NTI contre $28 \%$ de céramiques fines (parois épaisses et parois fines cumulées). À Mervent, la situation est exactement inverse, les céramiques fines sont les plus nombreuses avec $48 \%$ de l'effectif contre $30 \%$ de productions grossières.

- Corrélation groupes de pâtes/modes de cuisson

Aux Rogelins, cinq groupes de pâtes sur six sont majoritairement cuits en mode $\mathrm{B}$. Pour les argiles fines à parois fines, le mode A prédomine avec $55 \%$ des individus. Il est de $78 \%$ à Mervent.

\section{- Corrélation groupes typologiques/groupes de pâtes}

Pour le groupe typologique A des Rogelins (formes basses ouvertes), les argiles le plus souvent utilisées sont semi-fines à parois épaisses et grossières à parois fines. À Mervent, elles sont essentiellement fines et semi-fines. 

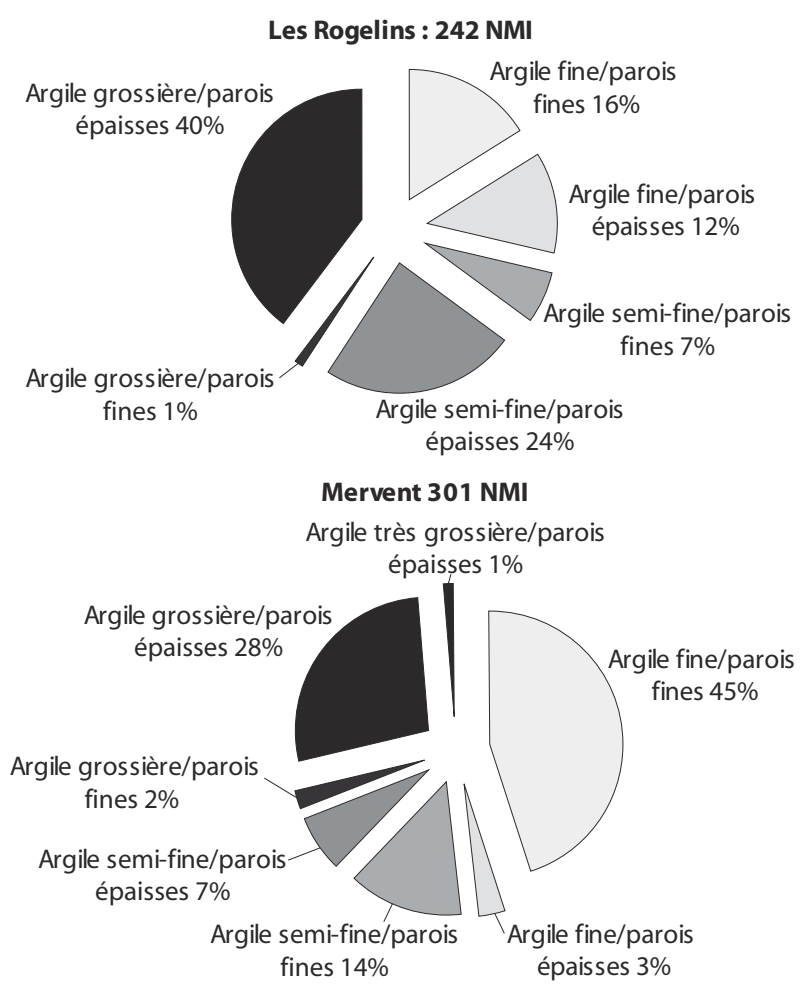

Figure 14 : Catégories des pâtes des céramiques aux Rogelins et à Mervent. DAO J.-M. Bryand.

Figure 14: Categories of clay of ceramic in Rogelins and in Mervent.

Avec le groupe typologique B (formes basses fermées), les proportions de céramiques fines et semi-fines des Rogelins progressent, elles sont inférieures à celles de Mervent.

Le groupe typologique $\mathrm{C}$ (formes hautes ouvertes) ne comporte que deux individus.

Le groupe typologique D (formes hautes fermées) des Rogelins utilise des argiles grossières à parois épaisses à $90 \%$. Ce taux n'est que de $50 \%$ à Mervent, mais les comptages ne concernent que 20 individus.

\section{Les décors}

\section{- Typologie}

Ajout de matière : les cordons rapportés

Les cordons, quand ils sont périphériques, se situent à un point d'inflexion du profil du vase, au niveau de l'épaule. Ils sont digités, l'empreinte du doigt est ovalaire, son axe est légèrement oblique (fig. $15: \mathrm{n}^{\circ} 1$ ). Dans un cas, des cordons pourraient se combiner de façon perpendiculaire sur la panse du récipient (fig. 15, $\mathrm{n}^{\circ}$ 2). Ce type d'ornementation se rencontre à la fin du premier Âge du Fer à Mervent (Nillesse et al., 2014). Une céramique haute fermée de la Croix de Laps à Civaux dans la Vienne comporte ce décor, elle est datée de La Tène ancienne (Pautreau et al., 1993).

\section{Ajout de matière : les moulurations}

Au moment du façonnage, le potier repousse la terre de façon à produire un relief. Il est très probable que le tour lent a été nécessaire pour réaliser les moulures (fig. 15, $\mathrm{n}^{\circ} 3$ ).

\section{Enfoncement de la matière au doigt ou au bâtonnet}

Ce procédé est appliqué sur la partie sommitale des lèvres et sur les panses.

Sur les lèvres, les dépressions obtenues tendent vers une forme ronde ou ovalaire (fig. 15, $\mathrm{n}^{\text {os }} 4$ et 5). Parfois les dépressions oblongues et obliques sur la partie sommitale de la lèvre semblent imiter la vannerie, les branches de saule torsadées (fig. 15, $\mathrm{n}^{\circ}$ 6).

Sur les panses, un registre de dépressions est appliqué, le plus souvent, au niveau de l'épaule. Les dépressions sont globalement ovalaires (fig. 15, no 11) ou allongées (fig. 15, $\mathrm{n}^{\circ} 12$ ). Elles sont espacées (fig. $15, \mathrm{n}^{\circ} 7$ ) ou rapprochées (fig. 15, no 8).

\section{Enfoncement de la matière : estampage}

Les motifs estampés sont obtenus par l'impression d'une étampe positive sur une pâte fraîche.

\section{Les motifs}

On distingue les ocelles qui peuvent être doubles (fig. 16, $\mathrm{n}^{\text {os }} 1$ à 3 ) ou triples (fig. 16, no 4), les croix de Saint-André (fig. 16, $\mathrm{n}^{\text {os }} 5$ et 6 ), les croissants de lune (fig. $16, \mathrm{n}^{\circ} 7$ ) et les points qui ont une forme oblongue (fig. 16, $\mathrm{n}^{\text {os }} 8,9,11$ ) ou quadrangulaire (fig. $16, \mathrm{n}^{\text {os }} 10$ et 12 ).

\section{Organisation des motifs et comparaisons}

Les ocelles sont regroupées en grappe (fig. 17, $\mathrm{n}^{\circ} 1$ ). Cette composition trouve d'assez nombreuses comparaisons. On la trouve par exemple, au Petit Cabaret et à la Renaîtrie (fig. 18) pour La Tène A2/B1, puis à La Tène $\mathrm{B}$ dans le Camp de Saint-Symphorien (Menez, 2008).

À la Renaîtrie (Poirier et al., 2005), les ocelles sont associées à des têtes d'oiseaux (fig. 18), on se situe probablement dans les premières utilisations du motif. Les formes céramiques du site présentent des gobelets à carène proéminente qui sont une évolution du mal nommé " gobelet jogassien ». Les pieds creux ne sont plus hauts comme au Hallstatt D3 et les lèvres des vases ne sont pas encore développées en véritables " amandes " comme à La Tène C. L'absence de caractères archaïques dans la céramique indique très probablement La Tène A2 ou le début de La Tène B1.

Le site de la Croix Boizard (Barbier, 1995) possède également des vases décorés d'ocelles groupées (fig. 18). Ils sont associés à une passoire exceptionnelle. La datation de l'objet caractéristique du Early Style (Gomez de Soto, 2005) se situe dans la seconde moitié du $v^{e}$ s. av. J.-C. ou au début du Iv ${ }^{e}$ siècle av. J.-C. Pour confirmer cette datation, il faut 
Chacé, les Rogelins : un site des débuts de La Tène en Anjou

157

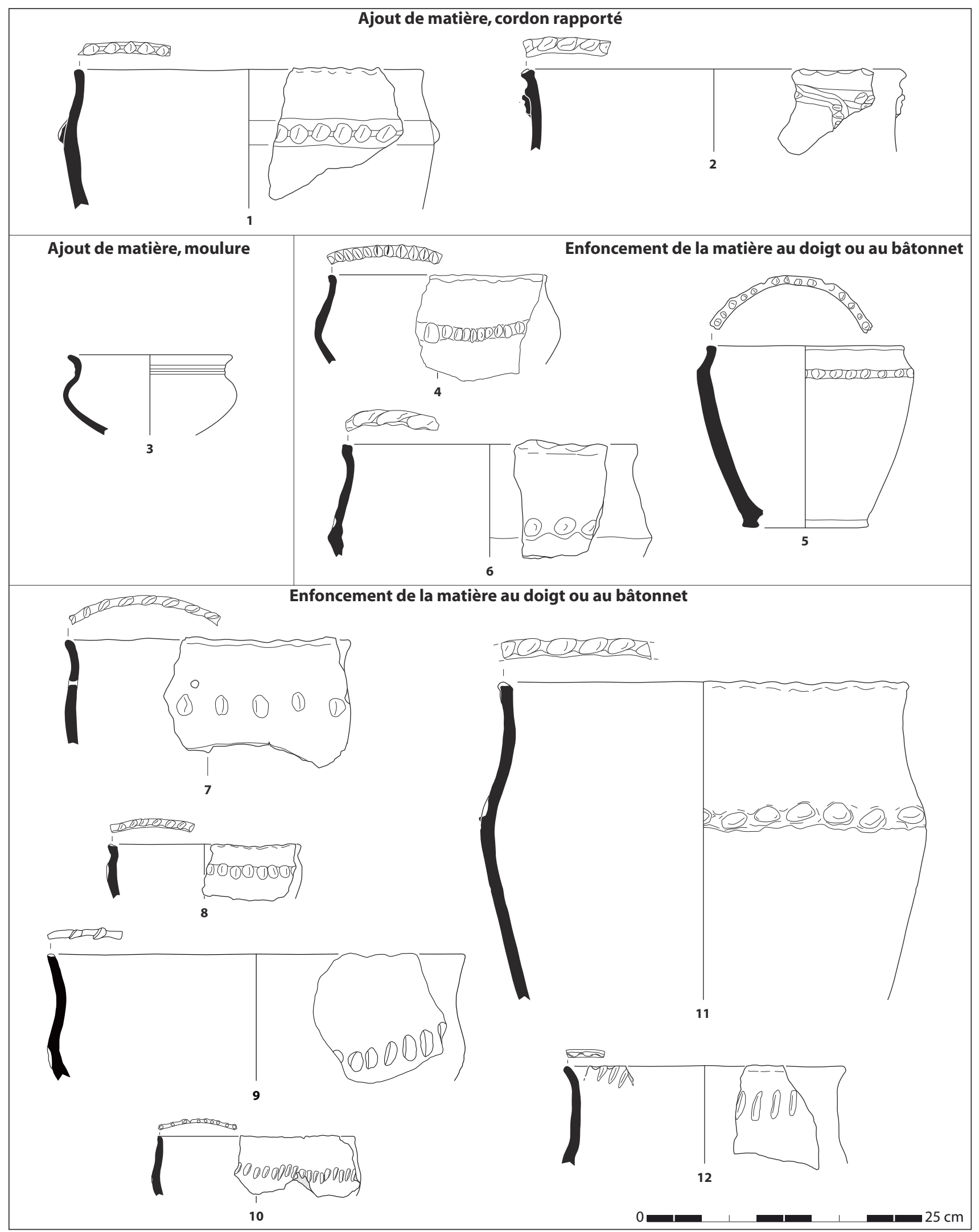

Figure 15 : Les décors plastiques. DAO J.-M. Bryand.

Figure 15: The plastic decorations. 
Enfoncement de la matière : estampage, les motifs

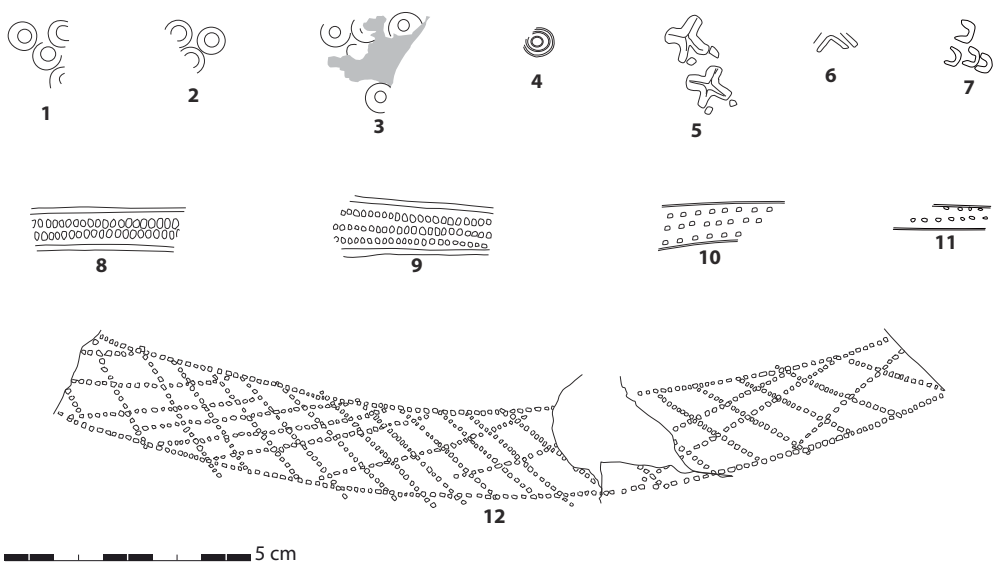

Figure 16 : Les motifs estampés des Rogelins. DAO J.-M. Bryand.

Figure 16: The motives used for stamped ceramics in Rogelins. prendre en compte, le tesson orné de deux ocelles reliées par des lignes courbes de points (fig. 18), qui annonce peut-être le style en arc de F. Schwappach (1969). Il ne prendra sa forme classique qu'à partir du IV $\mathrm{e}^{\mathrm{e}}$, puis au $\mathrm{III}^{\mathrm{e}}$ siècle av. J.-C. (Le Goff, 1992; Cherel, 1996). Régionalement, c'est le cas à la Chaîne (fig. 18) dans le Maine-et-Loire, site que l'on peut attribuer à la fin de La Tène B2, grâce à un fourreau d'épée orné (Nillesse, 2004).

$\mathrm{Au}$ Petit Cabaret, les ocelles sont disposées en grappe (fig. 18). La récente publication de ce site a parfaitement montré la similitude de son mobilier avec celui de la Croix Boizard. Les deux sites sont attribués à La Tène A2/B1 (Levillayer et al., 2013).

L'unique ocelle triple du site (fig. 17, $\mathrm{n}^{\circ}$ 2) est un motif récurent en Armorique, il en existe plusieurs attestations à Paule. Par contre, son association avec des lignes de points aux Rogelins encadrées d'incisions parallèles formant des bandeaux ne semble pas attestée dans le répertoire breton (Cherel, 1996). Il pourrait s'agir d'un trait stylistique local.

Les croix de Saint-André (fig. 17, ${ }^{\text {os }} 3$ et 4 ) sont des motifs fréquents en Armorique (Menez, 1996). Par contre, aux Rogelins, ils sont disposés à la verticale et sont encadrés de deux lignes incisées contenant des lignes de points, alors que dans le répertoire breton, l'organisation est généralement horizontale. Il existe un répertoire décoratif commun avec l'Armorique, mais la composition est originale.

Les croissants de lune sont encadrés par des lignes verticales, il semble y en avoir trois par registre (fig. 17, $\mathrm{n}^{\circ} 8$ ). Le décor est trop incomplet pour pouvoir être analysé correctement.

Les motifs de points encadrés de lignes incisées formant un bandeau constituent le procédé décoratif majoritaire sur les céramiques estampées des Rogelins. La première variante se caractérise par des points rapprochés disposés en lignes doubles ou triples. Sur le tesson $n^{\circ} 6$ (fig. 17), un bandeau horizontal s'articule sur un second bandeau légèrement oblique. Le fond $\mathrm{n}^{\circ} 7$ (fig. 17) est souligné par une série de trois incisions perpendiculaires à l'axe du vase. Un bandeau vertical s'appuie sur ces lignes, alors qu'un autre est divergent, comme pour la céramique précédente. L'exemplaire $\mathrm{n}^{\circ} 9$ (fig. 17) présente des lignes brisées, il appartient au même vase que le tesson $\mathrm{n}^{\circ} 10$ (fig. 17). Le motif incomplet est composé de bandeaux s'articulant presque perpendiculairement. Ce procédé pourrait correspondre à une composition aboutissant à des métopes bordées de bandeaux et vierges de décors. On retrouve peut-être ce système sur la panse $n^{\circ} 11$ et le fond $n^{\circ} 12$ (fig. 17) qui appartiennent au même vase. Là encore le fond est marqué par des lignes incisées sur son pourtour, elles contiennent des lignes de points. Un bandeau vertical trouve sa base sur ces lignes. On retrouve cette organisation sur le fond $\mathrm{n}^{\circ} 3$ (fig. 17), mais dans ce cas, les bandeaux verticaux encadrent des croix de Saint-André.

Pour la seconde variante, le procédé est le même, mais les points sont plus espacés. Sur le vase ${ }^{\circ} 13$ (fig. 17), quatre bandeaux forment un carré, les angles étant disposés verticalement et horizontalement. Sur un côté, on peut remarquer que les lignes des bandeaux se croisent. Le tesson étant incomplet, on ne détermine pas s'il s'agit d'un mode décoratif ou d'une liberté du potier. Sur la forme no 14 (fig. 17), ne figure qu'un bandeau horizontal. Le tesson $n^{\circ} 15$ (fig. 17) possède deux bandeaux qui comme pour les séries précédentes à points rapprochés ne sont pas strictement perpendiculaires.

Ces décors en bandeaux contenant des lignes de points ne trouvent pas de comparaisons exactes, pas même en Armorique. Par contre à la Croix Boizard, on retrouve le même principe décoratif, c'est-à-dire des bandeaux et des lignes de points (fig. 18). Contrairement aux Rogelins, les lignes sont plus souvent doubles et les poinçons semblent 


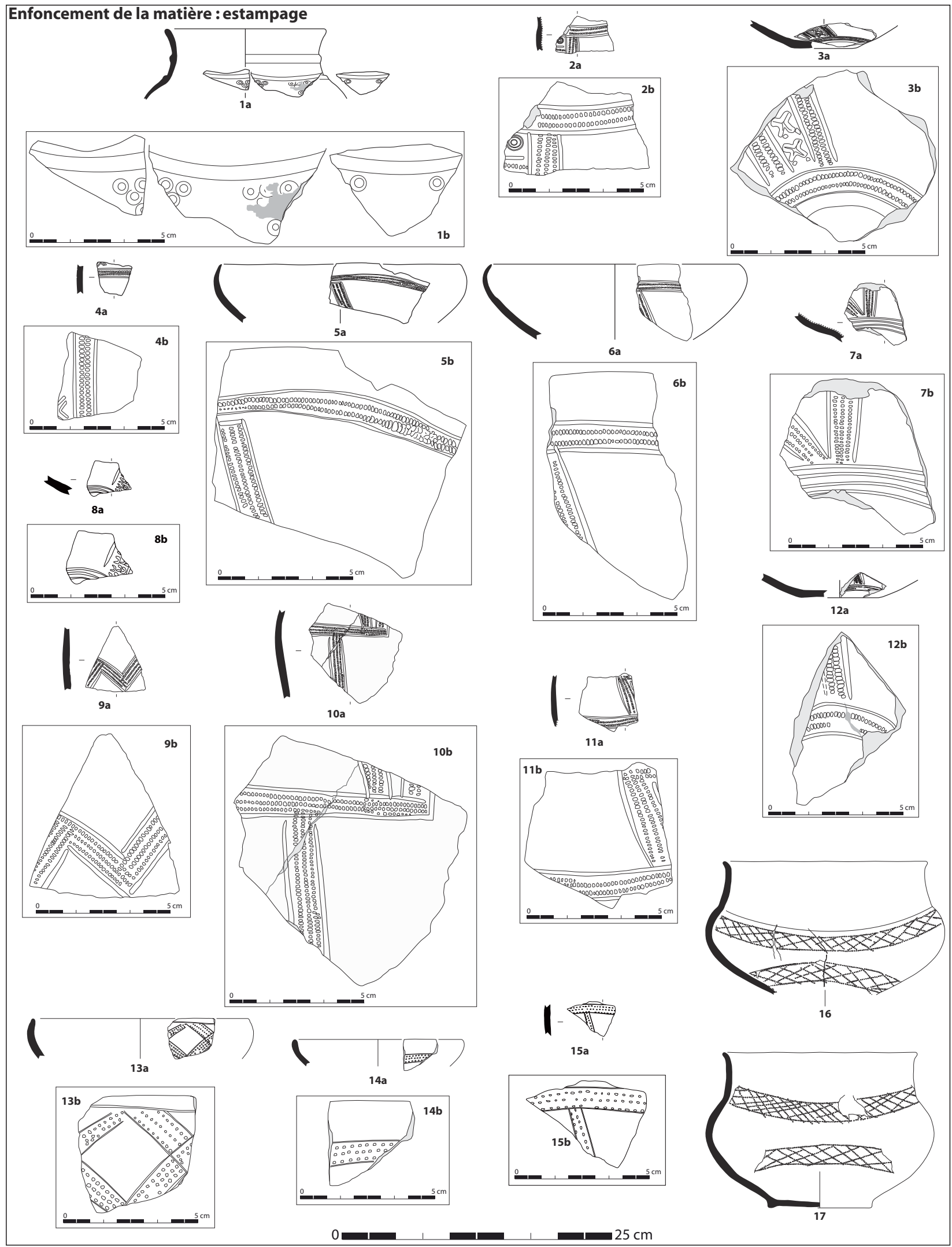

Figure 17 : Les décors estampés des Rogelins. DAO J.-M. Bryand. Figure 17: The stamped decorations in Rogelins. 


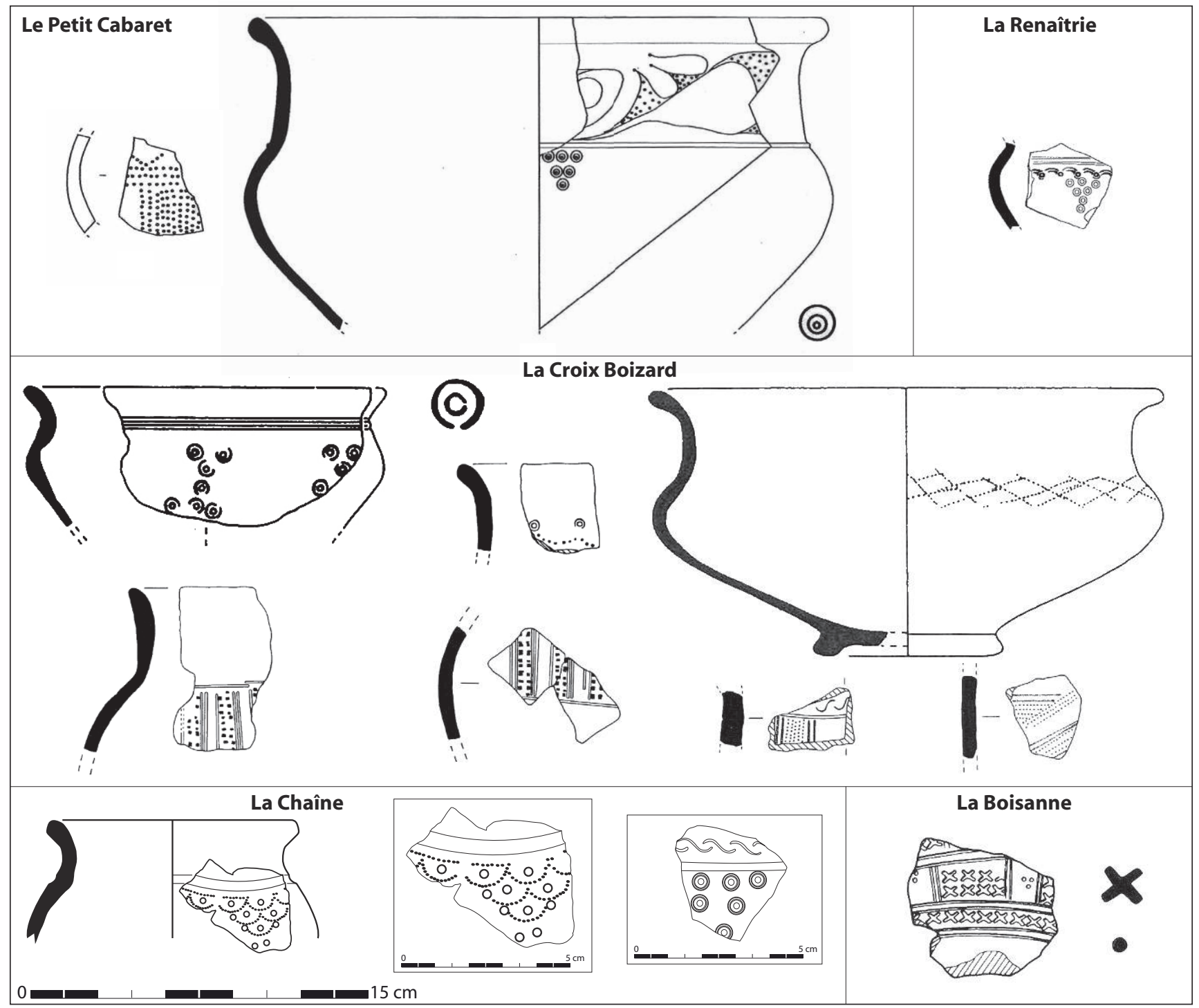

Figure 18 : comparaisons entre les céramiques des Rogelins, de la Croix Boizard (d'après Barbier 1995), de la Renaitrie (d'après Poirier et al., 2005), du Petit Cabaret (d'après Levillayer et al., 2013) de la Chaîne (d'après Nillesse, Bryand, 2000) et du Boisanne (d'après Ménez 1996). Figure 18: Comparisons between ceramic of Rogelins, la Croix Boizard (after Barbier, 1995), of la Renaîtrie (after Poirier et al., 2005), of Petit Cabaret (after Levillayer et al., 2013), of la Chaîne (after Nillesse, Bryand, 2000) and of Boisanne (after Ménez 1996).

comporter moins de points, ce qui expliquerait l'irrégularité du décor. La comparaison avec Brion est intéressante, elle pourrait montrer l'existence d'un style local et démarquer l'Anjou de l'Armorique.

Le dernier procédé consiste à utiliser des lignes de points, mais sans les encadrer de lignes incisées (fig. 17, $\mathrm{n}^{\text {os }} 16$ et 17) Des lignes de points se croisent de façon à former des losanges. Ils sont disposés sur deux registres, le premier à la naissance du col, le second vers le fond du récipient. La composition est limitée par des lignes également parallèles formées de points. Le potier a réalisé un travail assez approximatif, les losanges sont irréguliers et son poinçon a dépassé le cadre qu'il s'est initialement imposé. Ce type de décor trouve une comparaison à la Croix Boizard (fig. 18). Les losanges y sont également irréguliers, mais dans ce cas, il n'y a qu'un registre et le décor n'est pas limité par des lignes horizontales.

\section{Enlèvement de la matière : incisions}

Les incisions ont déjà été évoquées pour les motifs estampés, il s'agit d'en présenter un autre type. Le fond du vase $\mathrm{n}^{\circ} 1$ (fig. 19) comporte une série d'incisions réalisées sur pâte fraîche et organisées en une sorte de rosace. Quatre sont à peu près en arc de cercle, une autre est ovalaire, la dernière 


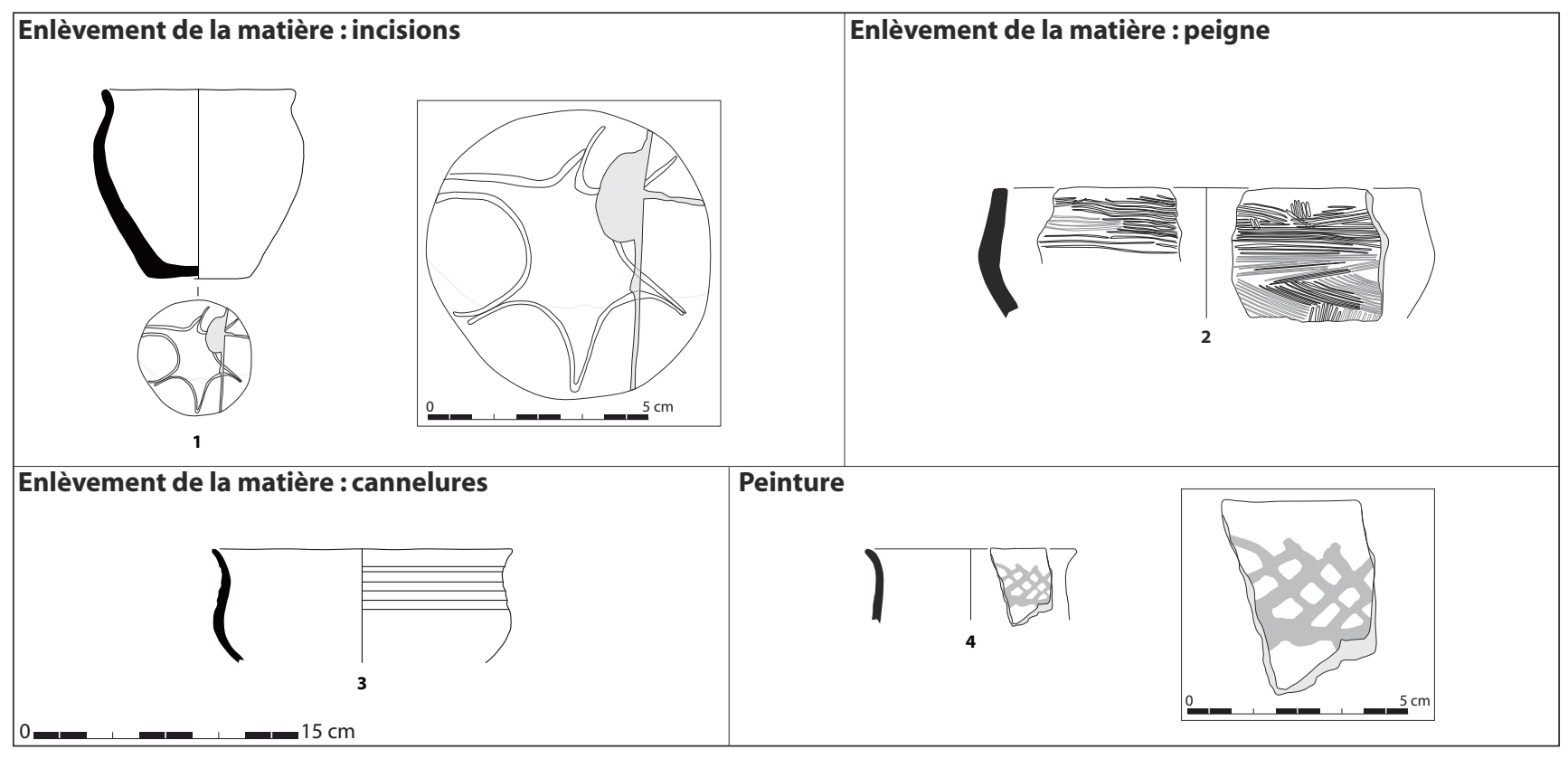

Figure 19 : Les décors : incisions, peigne, cannelures, peinture. DAO J.-M. Bryand.

Figure 19: Decorations: sections, comb, grooves, paint.

est irrégulière. L'ensemble forme une sorte d'étoile à six branches. Affirmer qu'il s'agit d'un décor n'est peut être pas exact : il n'est guère soigné et de plus, difficilement visible. Dans certains cas, comme dans les niveaux anciens de Paule dans les Côtes-d'Armor (Menez, 2008), on ne peut nier qu'il s'agisse d'une ornementation quand il s'agit de motifs poinçonnés réguliers et organisés. Dans d'autres cas comme au Boisanne dans le même département (Menez, 1996), on trouve des étoiles composées du croisement de lignes droites. La comparaison la plus probante est à chercher à Paule avec un motif formé de trois demi-cercles figurant un triangle aux côtés concaves. Quelle est la fonction de ce type d'incisions? Il ne semble pas assurer une meilleure stabilité au vase, l'hypothèse d'une marque de potier est envisageable.

\section{Enlèvement de la matière : peigne}

L'utilisation du peigne sur une céramique en cours de séchage peut émaner d'une démarche esthétique (fig. 19, $\mathrm{n}^{\circ} 2$ ), mais il est possible d'y voir aussi un intérêt pratique, le vase étant plus facile à saisir quand il est humide ou gras. L'usage du peigne est peu attesté aux Rogelins, alors qu'il est nettement plus fréquent dans des contextes de La Tène moyenne et finale.

\section{Enlèvement de la matière : cannelures}

Les cannelures constituent le seul décor de la série opéré par incision. Elles apparaissent sous la forme de bandes parallèles et régulières. Pour les réaliser, l'emploi du tour semble indispensable (fig. 19, $\mathrm{n}^{\circ}$ 3).

\section{Peinture?}

Le vase $n^{\circ} 4$ (fig. 19) présente la trace en " négatif " d'un motif de croisillons. C. Maitay qui a vu ce tesson a déjà observé un vase orné d'une peinture au graphite dont les fragments se sont conservés de façon différentielle. Le type de traces observables aux Rogelins permet de penser qu'il s'agit du fantôme d'une peinture graphitée (Maitay, 2007).

Les motifs peints au graphite sont très fréquents jusqu'au Hallstatt D3 dans le Centre-Ouest et ses marges, sauf vers le nord (Maitay, 2007; Gomez de Soto et al., 2007). Ils sont absents des Pays de la Loire et de la Normandie. À partir de La Tène ancienne, l'utilisation du graphite baisse sensiblement et il est utilisé pour réaliser des couvertes de peinture et non plus des motifs. C'est le cas à Paule (Menez, 2008). Un vase de la nécropole de Mazerolles dans la Vienne (Nicolini, 1983) est enduit de graphite, sa datation est à situer à la transition La Tène B/C. Un autre vase comporte à la fois un décor graphité et de la peinture rouge. L'association des deux enductions se développerait en Armorique à La Tène C (Daire, 1992). Enfin, un exemplaire est connu à Mervent pour La Tène C1. Le tesson des Rogelins est donc original.

\section{Corrélation type de décor/groupes de pâtes}

Il n'est pas étonnant de constater que les décors plastiques les plus fins (cannelures, moulures, estampages) apparaissent sur les productions réalisées en argile fine ou semi-fine. Sur les pâtes grossières, le dégraissant apparaissant souvent à la surface du vase, il n'est guère possible de les appliquer, les éléments contenus dans la terre pouvant être plus épais que 
le détail d'un motif. C'est également le cas de la peinture qui est difficilement applicable sur des surfaces rugueuses. À l'inverse, les décors les plus volumineux comme les digitations ou les impressions d'un bâtonnet et les cordons digités se posent sans aucune difficulté sur les pâtes grossières. L'utilisation du peigne se conçoit mieux sur une pâte grossière dont l'aspect de surface est souvent rugueux en raison de l'affleurement des dégraissants que sur une pâte fine dont l'épiderme est lisse. L'application du peigne sur ce type de pâte ruinerait le soin pris à utiliser une argile de qualité.

\section{Caractérisation et datation de la céramique des Rogelins}

Le nombre de restes (6138) est assez élevé par comparaison avec les autres séries régionales et le nombre typologique d'individus est remarquable avec un total de 270 formes de vases identifiées. Cela tient à des conditions taphonomiques particulièrement bonnes ( $\mathrm{du}$ moins pour la céramique). Le second aspect tient à la chronologie du lot, les sites de La Tène ancienne étant particulièrement discrets dans les Pays de la Loire. Ainsi, sur la carte archéologique de cette région, La Tène ancienne ne représente qu'un peu plus de $2 \%$ des entités archéologiques connues, alors que cette période couvre $40 \%$ de la plage chronologique occupée par le second Âge du Fer.

La céramique des Rogelins a été comparée avec celle de Mervent, habitat fortifié de hauteur attribué au Hallstatt D3. Bien entendu, les statuts des deux sites sont différents et l'usage des céramiques n'est peut-être pas le même sur les deux habitats. Néanmoins, on peut souligner certains aspects.

Concernant, la typologie, on remarque que le corpus des Rogelins contient plus de formes basses ouvertes (groupe A) que de formes basses fermées (groupe B), c'est l'inverse à Mervent. Cette évolution montre une préférence du potier et/ou du consommateur à La Tène ancienne vers les formes les plus simples à produire.

Pour la qualité des pâtes, on constate qu'elles sont plus grossières aux Rogelins qu'à Mervent et que parallèlement, le mode de cuisson B est largement majoritaire à La Tène ancienne aux Rogelins, alors qu'au Hallstatt D3, à Mervent, c'est le mode A qui prédomine.

Aux Rogelins, la quantification des types de décors montrent le quasi abandon des cordons rapportés digités, on lui préfère un motif plus rapide à mettre en œuvre, la digitation simple sur la panse. La nouveauté aux Rogelins, c'est l'apparition de l'estampage. Ce décor est également rapide à mettre en œuvre, la technique est efficace. Cependant, ce gain de temps n'est pas toujours synonyme de qualité, il suffit pour s'en convaincre d'observer l'irrégularité des compositions, le dépassement de la matrice hors du cadre devant être orné et le chevauchement des motifs. L'origine de l'estampage est à rechercher au premier Âge du Fer avec les étampes utilisées pour réaliser des cupules. Elles permettent de réaliser de profondes dépressions à la surface du vase. Le procédé nécessite une parfaite maîtrise de la plasticité de l'argile pour éviter une déformation générale de la forme lors de l'impression. En termes de production, son usage est certainement moins efficace que l'application d'un poinçon. La présence possible d'un tesson peint aux Rogelins étonne dans ce contexte chronologique. On aurait plus attendu des vases à couverte peinte et non un motif peint, cette céramique est peut-être plus ancienne que l'occupation principale du site.

Selon les critères utilisés au cours de cette étude, et selon des considérations modernes, on constate la moindre qualité des céramiques aux Rogelins par rapport à Mervent. Elle s'exprime par l'accroissement des formes les plus simples à réaliser dans des argiles souvent plus grossières.

Si la typo-chronologie de la céramique de l'Âge du Fer n'est toujours pas établie dans la région, le corpus des Rogelins est suffisamment documenté pour être daté. Deux tessons sont peut-être les plus anciens du site. Il s'agirait du goulot de la forme D60 et de l'exemplaire probablement peint au graphite. Néanmoins, la série ne présente pas de hauts pieds creux, ni de cupules qui disparaissent au tout début du second Âge du Fer. Il n'y a pas non plus de mal nommés "gobelets jogassiens ", qui dans leur forme classique carénée, marquent la fin du premier Age du Fer et le début du second (Nillesse et al., 2014). L'écuelle à profil en $S$ ne domine pas encore comme dans les séries les plus tardives de La Tène C, les lèvres des Rogelins n'ont d'ailleurs pas la forme en amande des productions de La Tène moyenne. La morphologie des vases de Chacé renvoie à La Tène ancienne; mais pas à ses prémices. Les comparaisons typologiques et/ ou les similitudes décoratives avec les ensembles de Brion, de la Renaîtrie et du Petit Cabaret, permettent une meilleure précision. Pour ces trois sites, il existe des vases ornés de motifs typiques du Early Style défini par P. Jacobsthal (1944) que l'on place entre la fin du $v^{e} s$. av. J.-C. et le début du IV siècle av. J.-C., c'est-à-dire à La Tène A2/B1.

Quatre datations par mesure du radiocarbone confirment cette attribution pour les Rogelins. F. 243 (Lyon 8719) se situe entre 751 et 402 cal. BC., F. 212 (Lyon-8718) entre 728 et 395 cal. BC. et F. 209 (Lyon-8717) entre 739 et 393 cal. BC. La datation de F. 207 (Lyon-8716) est un peu plus tardive : entre 703 et $368 \mathrm{cal}$. BC. Comme au Petit Cabaret (Lyon-8365 : entre 763 et 406 cal. BC.), on remarquera que la datation proposée pour le mobilier s'accorde seulement avec les dates les plus tardives fournies par le radiocarbone. 


\section{La faune}

L'ensemble étudié est composé de 2512 restes osseux (après remontage ${ }^{1}$ ), ramassés à vue, qui proviennent des niveaux de remplissage de 57 fosses. L'état de conservation des ossements est hétérogène. Des attaques naturelles liées à l'activité biologique et à la nature du sol ont pu être observées. Plus de $21 \%$ des restes déterminés présentent d'importantes concrétions qui ont rendu difficile, pour certains assemblages, les phases d'observations notamment celles concernant les traces anthropiques et les relevés d'usure dentaire. Ces agrégats incrustés sur la surface osseuse sont révélateurs de la présence d'humidité ou bien d'infiltration d'eaux de ruissellement au sein de ces structures

\section{- Le corpus}

Le taux de détermination aux rangs taxonomiques et anatomiques est de 52,8\% (tabl. 1). Le poids moyen des restes déterminés est de $21,9 \mathrm{~g}$, alors que celui obtenu pour les indéterminés est inférieur à $1 \mathrm{~g}$. Les espèces domestiques sont très nettement majoritaires avec 94,1\% du NR déterminé attribué aux cinq principaux taxons (NR5 = bœuf, mouton/chèvre, porc, cheval et chien). Les bœufs dominent très clairement l'ensemble que ce soit en nombre ou bien en poids de restes. Ainsi 52,1\% du NR5 est attribué aux bovins qui sont suivis par les caprinés puis par les porcs avec respectivement 31,6 et 12,8\% du NR5. Le cheval et le chien sont également présents, mais dans des proportions moindres avec seulement 1 et 2,5\% du NR5 déterminé. Si l'on considère les données en termes de poids des restes on obtient une image quelque peu différente. En effet, si le bœuf domine toujours l'ensemble avec près de $67,3 \%$ du PR5, il est suivi du cheval avec 19,3\%. Les caprinés et les porcs s'illustrent, quant à eux, par des proportions plus faibles mais relativement similaires avec respectivement 6,7 et $5,5 \%$ du PR5 ${ }^{2}$.

Il peut être mentionné que sur les 31 restes attribués au chien, neuf sont dispersés au sein des niveaux de comblement de cinq structures $(221,224,243,295,296)$ alors que 22 d'entre eux proviennent des niveaux de remplissage de la fosse 265. Diverses observations macroscopiques (distribution anatomique, latéralité et stade de croissance) indiquent un Nombre Minimum d'Individus de combinaison (NMIc) d'un chien pour cette fosse. Le cheval, quant à lui, se caractérise par un squelette en connexion anatomique (479 restes

1. Lors de ce comptage, les os qui s'avèrent, après remontage, n'appartenir qu'à un seul et unique os et dont la fragmentation est récente ont été comptabilisés comme un seul reste tout comme les squelettes et les parties anatomiques retrouvés en connexion anatomique.

2. Il est exact que le poids des restes est conditionné par la présence de concrétions sur certains ossements. Cependant, étant donné que celles-ci ont été observées indifféremment entre les espèces et les parties anatomiques, nous avons pris le parti d'utiliser ce type de décompte. pour un poids total de $4819 \mathrm{~g}$ ) issu de la fosse 234. Le poids moyen des restes de chevaux n'a pu être réalisé, la présence de cet individu en connexion aurait en effet faussé le résultat obtenu. L'ensemble de ses parties anatomiques est conservé (tête, côtes, vertèbres, ceintures, membres et bas-de-pattes) même les os de petite taille comme ceux des articulations ou de la queue (les carpes, les tarses et les vertèbres caudales). Ce qui indique que le cadavre a été déposé entier dans la fosse rapidement après la mort de l'animal, c'est-à-dire avant que la phase de décomposition ne commence (dépôt primaire). Les cassures fraîches ainsi que l'importance des concrétions grisâtres sur la totalité des ossements ont empêché la prise de mesures et l'observation de traces anthropiques. Au vu de ces éléments la distinction mâle/femelle n’a pu être réalisée. L'observation des stades d'épiphysation des os, selon les tables de Barone (1976) indique un âge à la mort compris entre 36 et 42 mois. Cette estimation est confortée et précisée par la présence de la deuxième incisive inférieure, ainsi que d'une prémolaire inférieure. Ces dernières étant en cours de renouvellement, elles indiquent un âge qui avoisine les 40-42 mois (Schmid, 1972).

Les taxons sauvages s'illustrent par 22 restes de mammifères ( 20 de cerf, 1 de chevreuil et 1 de lièvre), ils ne jouent donc qu'un rôle très modeste dans l'approvisionnement carné. Les restes attribués aux cervidés sont de deux types. Les premiers concernent les fragments de bois indéterminés $(\mathrm{NR}=14)$ qui informent du ramassage saisonnier de cette matière première et de son utilisation dans un cadre artisanal domestique. Un bois de massacre (F. 297) et des ossements appartenant aux membres et aux bas-de-pattes (F. 215 et F. 222) suggèrent davantage une activité de chasse, tout comme le métacarpe de chevreuil porteur d'un coup de tranchant (F. 243).

Pour les oiseaux, seul un humérus de gallinacé a été identifié (F. 227). Six autres restes d'oiseaux n'ont pu être identifiés au rang de l'espèce. Il peut être noté la présence non négligeable de 48 restes d'amphibiens pour un poids de $18 \mathrm{~g}$ (tableau 1). Les modes d'accumulation de ce type de faune sont toujours très difficiles à mettre en évidence, ceci d'autant plus lorsqu'aucune trace de découpe, de brûlure ou de digestion n'est observée. Le piégeage naturel apparaît alors une des hypothèses les plus plausibles.

- Sélection anthropique ou naturelle?

La distribution anatomique

Le poids des restes par partie anatomique permet de relativiser les données brutes qu'offrent les proportions des trois principaux taxons. Une méthode utilisant le poids des restes a été appliquée, à cet assemblage, afin d'essayer de mettre en évidence d'éventuelles sélections anatomiques d'ordre naturel ou anthropique (Lepetz et Oueslati, 2003). Elle consiste 


\begin{tabular}{|ll|rr|rr|r|}
\hline Noms vernaculaires & Noms scientifiques & NR & \%NR & PR (g) & \% PR & PM (g) \\
\hline Bœuf & Bos taurus & 650 & 52,1 & 18913 & 67,3 & 29,1 \\
Mouton/Chèvre & Ovis aries/Capra hircus & 394 & 31,6 & 1895 & 6,7 & 4,8 \\
Porc & Sus scrofa domesticus & 160 & 12,8 & 1605 & 5,7 & 10,1 \\
Cheval : & Equus caballus & 13 & 1 & 5438 & 19,3 & 7 \\
Chien & Canis familiaris & 31 & 2,5 & 271 & 1 & 8,7 \\
\hline Total mammifères domestiques & & $\mathbf{1 2 4 8}$ & $\mathbf{1 0 0}$ & $\mathbf{2 8 1 2 2}$ & $\mathbf{1 0 0}$ & $\mathbf{2 2 , 5}$ \\
\hline Cerf* & Cervus elaphus & 20 & & 966 & & \\
Lièvre & Lepus sp. & 1 & & 4 & \\
Chevreuil & Capreolus capreolus & 1 & & 7 & \\
\hline Total mammifères sauvages & & $\mathbf{2 2}$ & $\mathbf{9 7 7}$ & \\
\hline Coq & Gallus gallus & 1 & & 2 & & \\
Oiseau indéterminé & Aves sp. & 6 & & 3 & & \\
\hline Total oiseaux & & 4 & & 18 & \\
\hline Amphibien & Amphibia & 1326 & 52,8 & 29128 & 93,2 & 21,9 \\
\hline Restes déterminés & 1186 & 47,2 & 2126 & 6,8 & 0,8 \\
\hline Restes indéterminés & & $\mathbf{2 5 1 2}$ & $\mathbf{1 0 0}$ & $\mathbf{3 1 2 5 4}$ & $\mathbf{1 0 0}$ & $\mathbf{1 2 , 4}$ \\
\hline TOTAL & & & &
\end{tabular}

+1 reste humain, pour $6 \mathrm{~g}$

- dont 1 squelette en connexion anatomique (soit 479 restes, pour $4819 \mathrm{~g}$ )

* dont 1 bois de massacre et 14 fragments de bois (681 g)

à comparer les proportions en poids de restes de chacun des trois principaux taxons domestiques, par grande section anatomique, avec celles obtenues sur des squelettes de référence ${ }^{3}$.

Les proportions pondérales obtenues pour les bovins montrent que quatre des parties anatomiques sont surreprésentées (tête, scapulas, membres et bas-de-pattes) au détriment du squelette axial, vertèbres et côtes, qui ne s'illustre que faiblement. Seul le coxal est présent dans des proportions pondérales relativement proches de celles obtenues par le squelette de référence (fig. 20).

Pour les caprinés, les scapulas et les bas-de-pattes sont présents dans des proportions très proches de celles du squelette de référence (fig. 20). Les os de la tête et des membres, plus résistants, sont pondéralement surreprésentés alors qu'une nouvelle fois les côtes et les vertèbres, plus fragiles, présentent des proportions bien inférieures à celles du squelette de référence. Les petits ruminants paraissent ainsi avoir été plus touchés par les effets de la conservation différentielle.

La distribution anatomique des restes de porc présente des caractéristiques similaires en ce qui concerne les restes de la tête, des vertèbres, des côtes et des scapulas. À l'inverse, elle se différencie par une sous-représentation des coxaux, des membres et des bas-de-pattes (fig. 20). Ceci est intéressant, car que ce soit pour les porcs ou les petits ruminants, les basde-pattes sont sous-représentées alors qu'ils sont composés d'os pourtant résistants aux diverses attaques naturelles.

L'analyse de la distribution anatomique donne ainsi l'image de rejets mixtes, c'est-à-dire de déchets issus des phases de préparation (tête, bas-de-pattes, extrémités d'os longs), mais également de la consommation (ceintures, membres, vertèbres et côtes). Cependant, il est important de noter que quelle que soit l'espèce prise en compte, toutes les régions anatomiques sont présentes ce qui suggère que

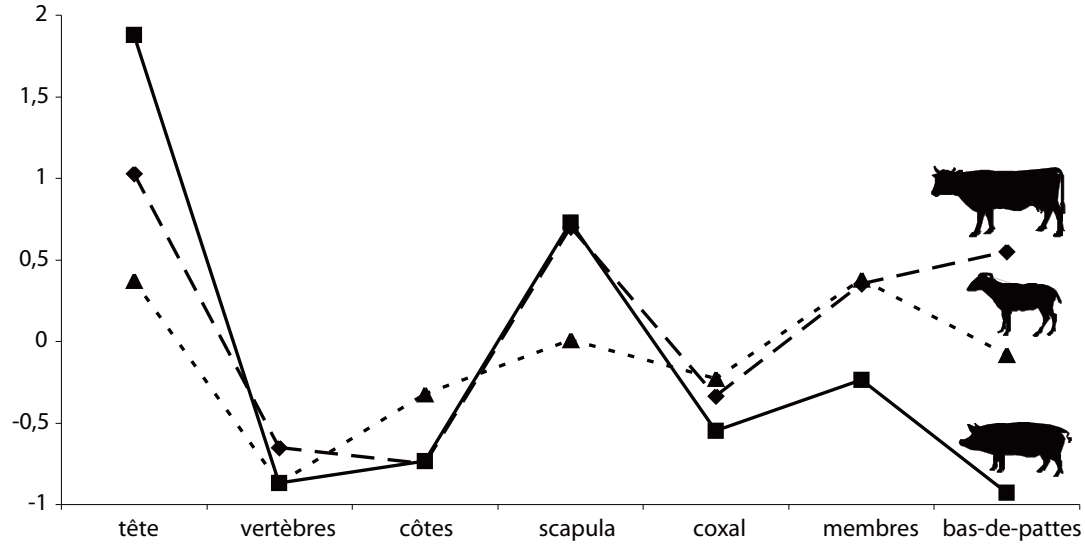

Ecart relatif des \% entre l'assemblage archéologique et le squelette de référence
Figure 20 : Proportions pondérales des différentes parties anatomiques des trois principaux taxons de la faune. DAO A. Baudry. Figure 20: Weight proportions of three main domestic species by body parts.

3. Les données de référence utilisées sont celles publiées par Lepetz et Oueslati (2003). 
ces individus ont vraisemblablement été élevés, abattus et consommés sur place ou à proximité.

\section{- Gestion des cheptels : l'âge au décès}

Quelques estimations des âges au décès ont été réalisées pour les trois principaux taxons domestiques ${ }^{4}$. Il faut toutefois garder à l'esprit que les données sont trop peu nombreuses pour permettre d'approcher les modalités de gestion du cheptel. La faiblesse quantitative des données a nécessité de travailler en nombre de restes et non en nombre minimum d'individus corrigé. Ces résultats doivent, par conséquent, être utilisés avec la plus grande prudence et ne renseignent qu'en termes de présence/absence.

Pour les bovins, il est important de signaler trois restes de métapodes attribués à des néonataux (F. 224 et F. 221). Cet élément indique l'existence de femelles en gestation sur le site ou à proximité. En ce qui concerne les données dentaires, seule une mandibule permet une estimation comprise entre un et deux ans, alors que sept autres indiquent des âges à la mort entre deux et quatre ans. Ces individus ont vraisemblablement été élevés pour la production de viande. On relève six bêtes dont l'âge est supérieur à six ans dont deux d'âge avancé, supérieur à onze ans. Celles-ci correspondent davantage à des reproducteurs, des vaches laitières ou bien des bêtes de somme réformées (tableau 2). Cette utilisation est également attestée par une lésion observée sur un métacarpe de bœuf (F. 262, fig. 21) Il s'agit d'une déformation du bord latéral de la partie proximale de l'articulation. Elle pourrait correspondre à une ostéo-arthrite déformante qui pourrait être la conséquence d'une surcharge et d'un excès de travail (Chaix et Méniel, 2001; Driesch, 1975).

Les caprinés présentent des données différentes. Plusieurs mandibules montrent un âge à la mort situé entre un et quatre ans, ce qui correspond très certainement à la production de viande. D'autres, au nombre de trois, permettent des estimations comprises entre huit et dix ans, ce qui indique sur le site, la présence d'animaux producteurs de lait, de laine et des reproducteurs qui sont vraisemblablement abattus après avoir été réformés (tabl. 2).

En ce qui concerne les porcs, les individus abattus entre six mois et deux ans, au nombre de sept, correspondent très probablement à la consommation de viande fraîche. Ceux abattus à maturité pondérale, c'est-à-dire, entre deux et trois ans, semblent plus particulièrement destinés à des préparations telles les salaisons. Enfin, les individus

4. Elles ont pu être réalisées à partir de l'observation des stades épiphysaires de certains ossements (Barone, 1976) et de l'usure dentaire. Le système d'encodage retenu est celui de Grant (1982) et les classes d'âge absolu ont été obtenues à partir des travaux de Ducos (1968) et Lepetz (1996) pour les bovins, de Payne (1973) pour les caprinés et de HorardHerbin (1997) pour les porcs.

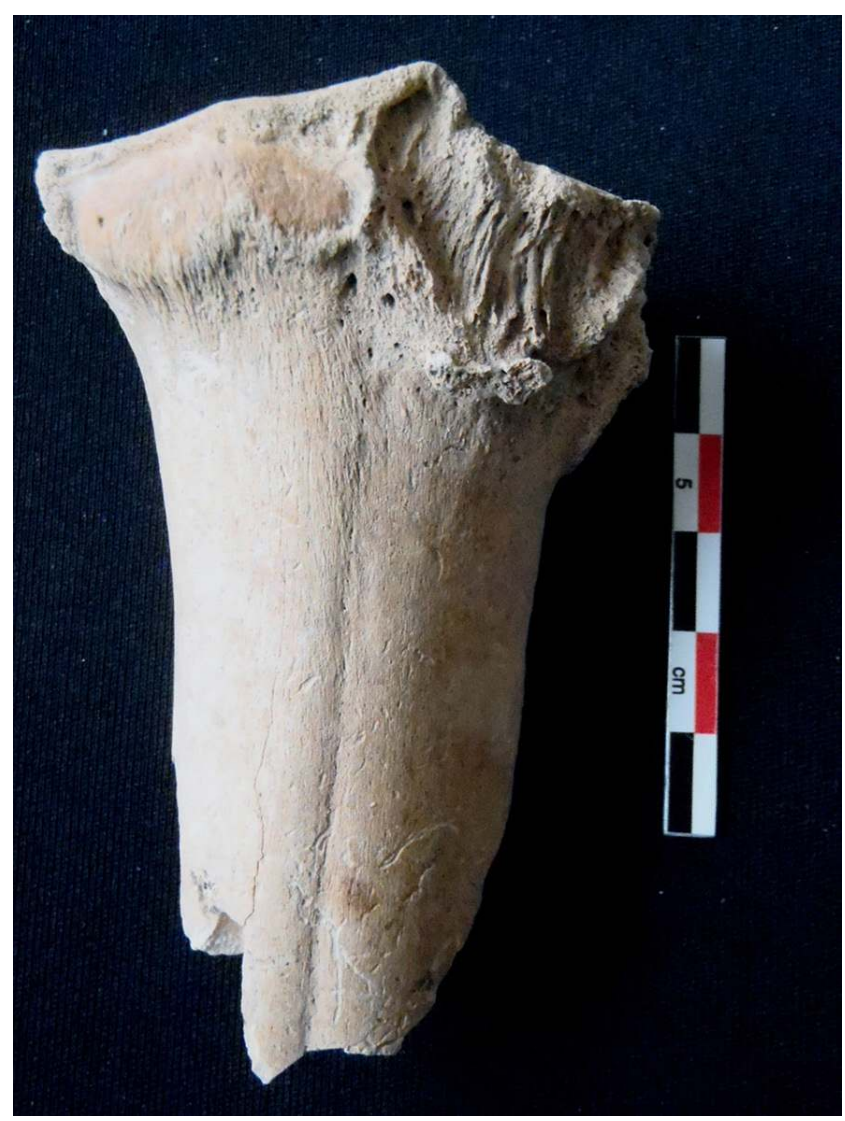

Figure 21 : métacarpe de bœuf, déformation du bord latéral de l'épiphyse proximale, F.262. Cliché A. Baudry.

Figure 21: Deformation of the proximal epiphysis on a Bos metacarpal, F. 262.

\begin{tabular}{|c|c|c|c|c|c|}
\hline Âge estimé & Effectif & Âge estimé & Effectif & Âge estimé & Effectif \\
\hline $0-1$ an & 0 & $0-6$ mois & 0 & $0-6$ mois & 1 \\
\hline $1-2$ ans & 1 & 6-12 mois & 1 & $6-12$ mois & 3 \\
\hline $2-4$ ans & 7 & $1-2$ ans & 10 & $12-18$ mois & 1 \\
\hline 4-6 ans & 0 & 2-4 ans & 5 & $18-24$ mois & 2 \\
\hline 6-9 ans & 2 & 4-6 ans & 0 & $24-30$ mois & 3 \\
\hline $9-11$ ans & 2 & 6-8 ans & 0 & 30-36 mois & 1 \\
\hline$>11$ ans & 2 & 8-10 ans & 3 & $36-60$ mois & 1 \\
\hline \multicolumn{2}{|r|}{$\mathrm{a}$} & & $\mathrm{b}$ & $>60$ mois & 1 \\
\hline
\end{tabular}

Tableau 2 : Estimations des âges à la mort à partir des données dentaires (en NR); a-Bœuf, b-Caprinés, c-Porc. Tableau A. Baudry. Table 2: Estimated mortality age distribution (NR); a-Bos, b-caprinae, c-Sus.

de plus de 36 mois sont conservés pour la reproduction (tabl. 2). Comme pour les bovins, un humérus et un métapode (F. 224) âgés de quelques semaines semblent correspondre à un cas de mortalité indissociable de toute activité d'élevage. 
- Consommation carnée et ressources animales : les traces anthropiques

L'observation des traces anthropiques a été fortement limitée par des concrétions qui recouvrent, dans certains cas, une très grande partie des surfaces osseuses. Quelques traces de découpe, environ soixante-dix, ont toutefois été mises en évidence. Elles sont plus particulièrement visibles sur les os de bœuf ( $\mathrm{NR}=43$ ) et de caprinés ( $\mathrm{NR}=22)$, les porcs quant à eux présentant très peu de stigmates $(\mathrm{NR}=7)$. Malgré leur nombre relativement limité, elles sont réparties sur l'ensemble des parties anatomiques et illustrent différentes étapes, de la préparation de la carcasse jusqu’à la consommation. Aucune trace de mise à mort des animaux n'a été observée. L'étape de séparation de la tête est ici attestée par plusieurs mandibules de bœuf $(\mathrm{NR}=6)$ et de porc $(\mathrm{NR}=2)$ sectionnées au niveau de la branche mandibulaire. La désarticulation des pieds, le dépeçage et le prélèvement de tendons sont, quant à eux, miss en évidence par des traces fines sur les phalanges proximales (bœuf $N R=1$ ), les tarses (bœuf NR $=2$ ) et les métapodes (bœuf NR = 1 et caprinés $\mathrm{NR}=1)$.

Les vertèbres et les côtes non fragmentées sont peu nombreuses, il est alors difficile d'aborder l'étape du partage de la carcasse. Quelques traces fines visualisées sur une dizaine de côtes de bœuf et une côte de porc illustrent le prélèvement de la viande. La séparation de la carcasse en quartiers de viande est visible par le biais de plusieurs types de traces sur les ceintures et sur les membres. Cette pratique est lisible sur deux coxaux de bœuf. Des épines scapulaires tranchées et des traces parallèles, laissées par le couteau, sur quatre scapulas de bœuf et une de caprinés témoignent vraisemblablement du prélèvement de morceaux de viande : macreuse et jumeau (Bellanger et al., 2014). Une trace circulaire observée sur une scapula de bœuf peut probablement être attribuée à la suspension de la pièce de viande à un croc de boucher (HorardHerbin, 1997). La séparation des carcasses et du traitement des membres est également visible des coups de tranchants localisés au niveau des os longs des membres, mais aussi des métapodes. Ils ont pu être observés sur des restes de bovins (trois humérus, un radius, deux fémurs, quatre tibias, deux métacarpes et six métatarses), de caprinés (cinq radius, un fémur, sept tibias, trois métacarpes et trois métatarses) et de porc (trois humérus). Les enlèvements d'éclats sur les diaphyses des os longs (bœuf, $\mathrm{NR}=3$ et caprinés, $\mathrm{NR}=2$ ) indiquent, quant à eux, la décarnisation.

Aucune trace de découpe n'a été observée sur les restes de cheval. En ce qui concerne le chien, il est intéressant de noter que quelques-uns des restes mis au jour au sein de la fosse 265 sont porteurs de traces anthropiques. Ainsi des traces fines situées sur le bord articulaire de l'atlas indiquent la séparation du crâne et de la première vertèbre cervicale alors que plusieurs traces réalisées au couteau sur les zones articulaires proximales et distales illustrent la désarticulation des membres. Enfin, peuvent être mentionnées des traces qui sont davantage à associer à l'utilisation des matières animales à des fins artisanales. Il s'agit d'entailles régulières visualisées à la base d'une cheville osseuse de bœuf (prélèvement de l'étui corné?) et de bois de cervidés (F. 297) portant des traces de découpe.

\section{- Discussion et comparaisons}

La liste de faune est très nettement dominée par les trois taxons domestiques. Ce sont toutefois les bœufs qui paraissent fournir l'essentiel de l'apport en protéines animales. Les caprinés semblent jouer un rôle secondaire, alors que les porcs ne sont présents qu'en très faible proportion (fig. 22). Les espèces sauvages ne prennent part que de façon anecdotique à l'approvisionnement carné du site. Ceci est toutefois un élément récurrent au sein des assemblages archéozoologiques des habitats de l'Âge du Fer, qui présentent des spectres de faune sauvage peu diversifiés ne dépassant que très rarement les $3 \%$ du nombre de restes déterminés (Méniel et al., 2009). Les bovins semblent donc être l'espèce privilégiée ce qui pose une nouvelle fois la question des spécificités de l'élevage dans l'Ouest de la France pour la période de l'Âge du Fer. Au niveau régional, les sites contemporains de la Croix Boizard à Brion (Yvinec, 1995, cité in Méniel et al., 2009), de la Fauche Verdon à Luigné (Borvon, in Levillayer et al., 2013) et du Petit Cabaret à Cizay-la-Madeleine (Borvon, in Levillayer et al., 2013) présentent de nombreuses caractéristiques similaires à celles énoncées tout au long de cette étude. Ainsi sur le site de la Croix Boizard, le type de structures concernées (fosses), le nombre total de restes $(\mathrm{NR}$ total $=2468)$, les proportions de bœuf $(52,8 \%$ du NR5) ainsi que la très faible diversité et proportion des taxons sauvages $(99,5 \%$ des restes sont attribués aux NR5 et seuls deux restes de cerf ont été identifiés), sont extrêmement proches de ceux mis en évidence sur le site de Chacé. Le bœuf arrive également en première position sur les sites de la Fauche Verdon et du Petit Cabaret, les proportions sont toutefois moindres avec respectivement $47 \%$ et $32 \%$ des principaux taxons domestiques (fig. 26). Les espèces chassées sont également numériquement faibles, sur ces deux sites, car elles ne représentent que 2,1\% des restes au Petit Cabaret et 3,4\% à la Fauche Verdon (Levillayer et al., 2013). La diversité des taxons sauvages est cependant plus grande qu'aux Rogelins avec la présence du sanglier et du campagnol à la Fauche Verdon, du blaireau et de la taupe au Petit Cabaret (Levillayer et al., 2013).

À une échelle plus large, les données obtenues pour le Grand-Ouest présentent également des données similaires. Ainsi, si les sites attribués à La Tène ancienne présentent de 
Figure 22 : Proportions relatives des trois taxons domestiques, en nombre (NR3 = 1204) et en poids (PR3 $=22413 \mathrm{~g}$ ) de restes. DAO A. Baudry. Figure 22: Frequency of the three main domestic species by number $(N R 3=1204)$ and weight $(P R 3$ $=22.413 \mathrm{~g}$ ) of bones.

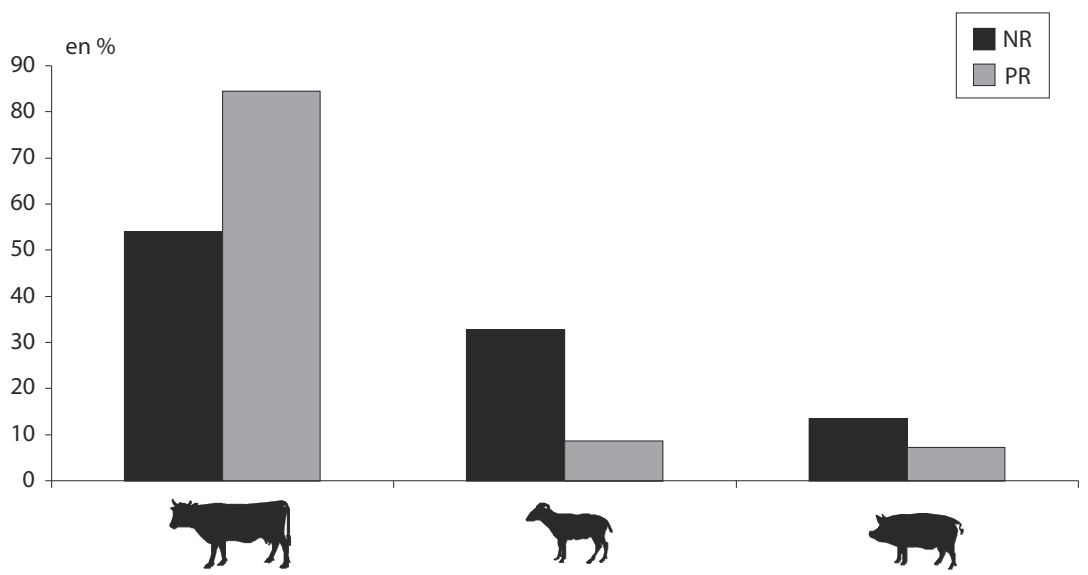

façon récurrente des assemblages peu volumineux (Auxiette et al., 2010), quelques sites contemporains ont toutefois fait l'objet d'études archéozoologiques ces dernières années en Basse-Normandie et plus particulièrement dans le Calvados. Les sites de Fierville-les-Parcs, le Pré de la Val (Méniel, in Jahier et al., 2002), de Courseulles, la Fosse Touzé (Yvinec, in Jahier et al., 2011), de Soulangy, RD68 (Méniel cité in Auxiette et al., 2010), d'Eterville, les Prés du Vallon (Baudry, in Giazzon et al., 2009) et de Condé-sur-Ifs, la Bruyère du Hamel (Baudry, in Dron et al., 2010) apportent de précieux éléments comparatifs. Ces analyses archéozoologiques présentent systématiquement des proportions très élevées de bovins (fig. 23). Il est également très intéressant de noter que cet élément est systématique, et que ces restes proviennent de niveaux de comblement de fosses ou bien de fossés. Cette distribution n'est donc pas uniquement à rattacher aux effets de la conservation différentielle, mais plus vraisemblablement à de réels choix de gestion.

Ces divers éléments se différencient très nettement de ceux observés pour l'Est et le Nord de la France, zones géographiques pour lesquelles ce sont les petits ruminants et les suidés qui dominent les spectres de faune au cours de La Tène ancienne (Méniel et al., 2009). Cette tendance apparait ainsi caractériser une zone comprenant les actuelles régions de Basse-Normandie, de Bretagne et des Pays de la Loire et persister tout au long de l'Âge du Fer (Auxiette et
Figure 23 : Fréquence relative des restes des trois principaux taxons domestiques, La Tène ancienne (comparaison avec les données de A. Borvon, P. Méniel, J.-H. Yvinec et A. Baudry citées in Méniel et al., 2009; Auxiette et al., 2010 ; Baudry, 2012 et Levillayer et al., 2013). DAO A. Baudry.

1-Courseulles, La fosse Touzé; 2-Chacé, Les Rogelins; 3-Cizay-la-Madelaine, Le Petit Cabaret; 4-Condé-sur-Ifs, La Bruyère du Hamel; 5-Soulangy, RD68; 6-Luigné, La Fauche Verdon; 7-Courseulles, La fosse Touzé; 8-Eterville, Les Prés du Vallon; 9-Brion, La Croix Boizard; 10-Fierville-les-Parcs, Pré de la Val.

Figure 23: Frequency comparison of the rests of three main domestics species. La Tène ancienne (comparison with the data of $A$. Borvon, P. Méniel, J.- $H$. Yvinec and A. Baudry, indicated in Méniel et al., 2009; Auxiette et al., 2010; Baudry, 2012 and Levillayer et al., 2013).

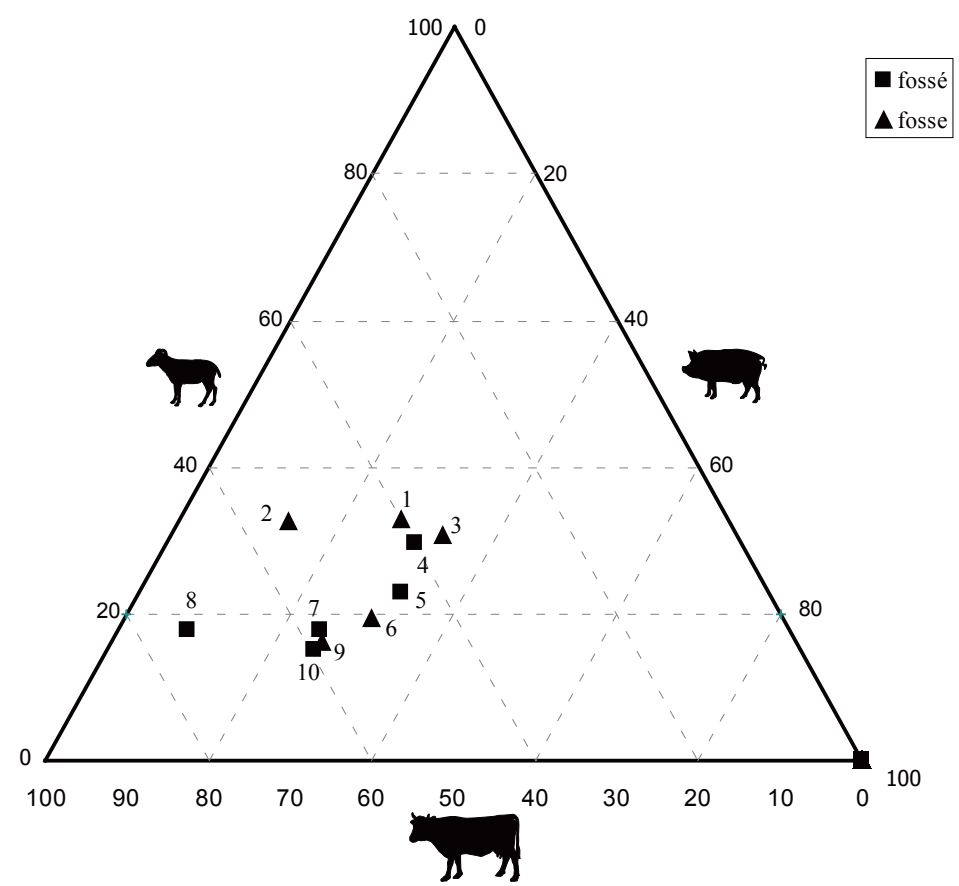


al., 2010; Baudry, 2012; Méniel et al., 2009; Germinet, 2009). Il est également important de noter que, sur le site des Rogelins, les trois principaux taxons domestiques et tout particulièrement les bœufs sont représentés par l'ensemble des parties anatomiques. Ces rejets illustrent les diverses phases de préparation des carcasses jusqu'à la consommation proprement dite. Les quelques estimations d'âge à la mort vont également dans ce sens avec la présence sur ce site de restes attribués à différentes classes d'âge. Même si les restes attribués au porc et aux petits ruminants ont été plus fortement touchés, par les effets de la conservation différentielle, ils reflètent néanmoins des comportements similaires.

\section{Le crâne humain du silo 214}

Le fragment crânien étudié correspond à un frontal quasi complet, ayant appartenu à un individu de taille adulte dont l'âge au décès et le sexe ne peuvent être estimés (fig. 24). Les processus zygomatiques sont absents ainsi que le toit des orbites. L'arcade sourcilière est assez marquée. Les bords pariétaux droit et gauche sont tous deux brisés. Les autres restes se rapportent vraisemblablement au même individu. Un élément de l'orbite est identifiable. Des fragments des deux processus mastoïdes, d'un fragment de bosse pariétale ainsi que des parties du maxillaire suggèrent un bloc craniofacial et non un masque humain comme cela a pu être constaté parfois à l'exemple de Montmartin (Boulestin et Duday, 1997) ou Gournay-sur-Aronde (Poplin, 1985). La

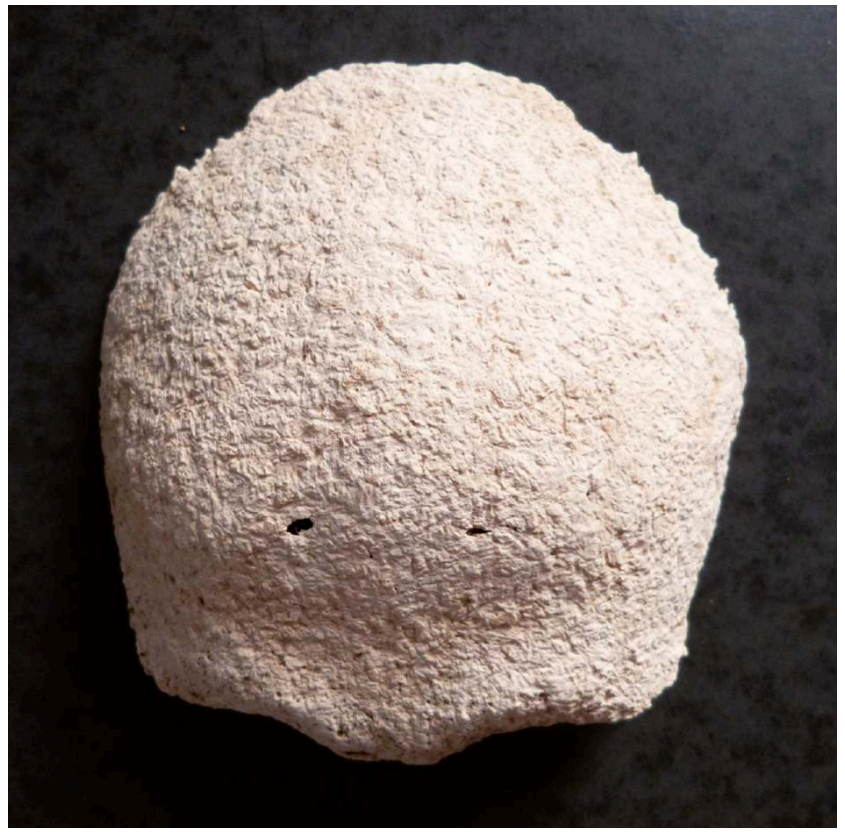

Figure 24 : Frontal humain des Rogelins, face exocrânienne. Cliché E. Rousseau.

Figure 24: The human frontal bone from the archeological site of les Rogelins, external surface. surface osseuse de l'écaille frontale ou corticale est très érodée à la fois sur la face endocrânienne et sur la face exocrânienne. Les conditions taphonomiques d'ensevelissement de la pièce expliquent pour partie l'état de dégradation importante de la surface osseuse du frontal. Toutefois, il est possible que cet état soit d'abord lié à une exposition du crâne aux intempéries de toutes sortes. Il porte une érosion très marquée identique à celle décrite pour certains os de faune. En effet, la conservation est très hétérogène, car de nombreuses concrétions les recouvrent (cf. analyse de la faune par A. Baudry). Cette destruction importante de la surface corticale interdit d'observer des traces de coupes ou de découpe de nature anthropique et de déterminer si ce crâne a fait l'objet d'une quelconque préparation (écorchement, coups, perforation...), comme cela a été démontré sur d'autres sites (Boulestin et Duday, 2012).

La mise au jour de restes humains et plus particulièrement crâniens dans les contextes archéologiques laténiens est maintenant un fait bien établi et connu des protohistoriens (Méniel, 1989; Rousseau, 2012). De manière générale, leur présence dans le comblement des structures fossoyées s'explique soit par un dépôt volontaire dans une structure donnée et choisie à cet effet, soit par des rejets dans une structure (silo, fossé, fosse, puits, trou de poteau) dont la fonction initiale est abandonnée. Le plus souvent le comblement détritique de ces creusements divers livre du mobilier archéologique de tout ordre parmi lequel figurent des os humains au même titre que les restes de faune consommée. Ces os sont aussi bien des éléments céphaliques que des os du squelette post-crânien. Toutes les classes d'âge peuvent être représentées. Certains os portent la trace manifeste de modifications de surface évoquant des coups ou des manipulations anthropiques attestant dans certains cas le démembrement des corps. Longtemps, ces découvertes ont été interprétées comme les vestiges de têtes coupées exposées ou comme la trace résiduelle de sépultures anciennes perturbées. La découverte de grands ensembles gaulois à caractère sacré comme à Gournay-sur-Aronde dans l'Oise, Ribemont-sur-Ancre dans la Somme, mais aussi d'habitats privilégiés comme à Montmartin dans l'Oise ont révélé à leur tour l'extraordinaire panel des manipulations et traitements subis par les corps réduits à l'état de cadavre. De plus, les multiples découvertes aujourd'hui bien documentées de sépultures en silo révèlent elles aussi l'étendue et la complexité des pratiques cultuelles ou funéraires de la période gauloise (Delattre, 2000; Bonnabel, 2010).

L'usage de couper les têtes chez les Gaulois est aujourd'hui bien attesté tant par les témoignages littéraires que par les découvertes archéologiques et les données anthropologiques nombreuses et bien documentées (Rousseau 2010; Rousseau 2012; Boulestin et Duday, 2012). Dans le cas de Chacé, 
l'hypothèse d'une tête trophée longuement exposée puis abandonnée ou rejetée après la chute de son support dans un silo devenu un dépotoir apparaît comme une hypothèse vraisemblable. Toutefois, il ne faut pas pour autant exclure d'autres interprétations possibles, par exemple qu'il s'agisse d'une relique, le crâne d'un ancêtre, conservée au sein de l'habitat. Les réouvertures de tombes à inhumation de LTA où l'extrémité céphalique est souvent absente car prélevée en témoignent (Rousseau, 2012). Les deux hypothèses sont plausibles mais rien ne permet de pouvoir privilégier l'une aux dépens de l'autre.

\section{Les restes carpologiques}

L'étude carpologique repose sur 25 prélèvements de sédiment issus de 17 silos se concentrant plus particulièrement au nord-ouest du site. L'extraction des restes carpologiques a été réalisée par flottation manuelle sur deux tamis de maille $2 \mathrm{~mm}$ et $0,5 \mathrm{~mm}$, puis par un tri sous loupe binoculaire. Les identifications s'appuient sur une collection de référence composée de semences actuelles ainsi que sur des atlas (Cappers et al., 2006; Jacomet, 2006; Beijerinck, 1976). La nomenclature (noms latin et vernaculaire) repose sur la flore électronique Tela Botanicas.

Enfin d'évaluer la proportion relative des différents taxons et des catégories végétales, le nombre de restes fragmentés de grains de céréales, de bases de glume, les cotylédons de légumineuses, de paléosemences sauvages est divisé par deux; les fragments de légumineuses et de coquille de fruits sont divisés par quatre. Les nombres obtenus sont ajoutés au nombre d'individus entier, il en résulte un nombre de restes corrigé (NRC).

\section{- Composition des assemblages}

À l'exception d'un échantillon stérile (F. 213, Us 4), les prélèvements livrent un total de 2838 carporestes majoritairement conservés par carbonisation. L'état de conservation est altéré par un encroûtement sédimentaire et une fragmentation importante. Onze paléosemences sauvages, essentiellement des Chenopodiacées, sont conservées par minéralisation (tableau 3), certainement du fait du substratum calcaire (Green, 1979).

Les données proviennent d'assemblages ouverts, c'est-àdire de carporestes d'origines diverses accumulés dans les structures. La majorité est caractérisée par des densités faibles (19 prélèvements), ces échantillons fournissent des informations sur les espèces les plus fréquentes, ils permettent donc de définir les productions agricoles et l'importance relative des espèces dans l'économie végétale.

5. [http://www.tela-botanica.org/site:botanique].
Dans cinq prélèvements les densités sont moyennes à fortes, ces assemblages sont également composés d'éléments hétérogènes (tableau 4) : balle $e^{6}$ de blés vêtus et d'orge, grains de blés nus et de millet commun, ainsi que de mauvaises herbes en proportions variables. Parmi elles, les messicoles sont majoritaires, devant les plantes de semis de printemps ou de cultures sarclées. Parmi ces dernières plusieurs sont des plantes rudérales très communes dans les zones anthropisées comme la morelle noire ou le chénopode blanc.

Pour les blés vêtus, la prédominance des éléments de l'épi sous la forme de bases d'épillet et de bases de glumes, parfois associés à de nombreuses mauvaises herbes, et à l'inverse l'absence de paille, indiquent qu'il s'agit de déchets d'une étape avancée de nettoyage des récoltes liée au décorticage des grains. Un résidu de décorticage de l'orge vêtue est également détecté dans le remplissage d'un silo 243 (Us 7) grâce à une concentration d'articles de rachis.

Les résidus de décorticage et de façon générale, la fréquence de la balle dans l'ensemble des échantillons indiquent que cette activité était récurrente sur le site. Plusieurs arguments plaident en faveur d'un traitement des blés vêtus en fonction des besoins : la fréquence de ce type de déchet en contexte d'habitat lors des périodes anciennes (Bouby, 2003); la pénibilité et la durée du décorticage révélées par les enquêtes ethnographiques (Hillman, 1981; 1984); les techniques de conservation qui favorisent un stockage avant décorticage; la conservation des enveloppes protègent les grains des ravageurs et de la moisissure (Sigaut, 1981).

Par conséquent, le décorticage des céréales vêtues tient une place importante dans les activités domestiques. Cette opération a pu être réalisée au mortier et au pilon, dont les expérimentations ont mis en évidence l'efficacité, notamment par rapport aux meules à va-et-vient (Meurers-Balke et Lüning, 1992).

- Les productions agricoles et la cueillette

Au sein des productions agricoles, les céréales sont prépondérantes. L'importance de chacune est évaluée en fonction de sa fréquence d'attestation, c'est-à-dire du nombre d'échantillons dans lequel une espèce est attestée. La céréaliculture repose en particulier sur l'amidonnier, le millet commun, l'engrain et l'orge vêtue. Cette dernière est certainement à rattacher à l'orge à quatre rangs dont neuf bases de lemmes sont attestées dans le silo 243 (Us 7, fig. 25). Cette orge est

6. Dans cette contribution on entend par balle l'ensemble des éléments de l'épi. Pour les blés vêtus qui se séparent en épillets après battage, la balle correspond plus particulièrement aux bases de glume, éléments les plus résistants des enveloppes et par conséquent les plus fréquemment attestées dans les prélèvements archéologiques, et aux bases d'épillet (bases de glume en connexion avec à l'article de rachis). Pour les blés nus et l'orge vêtue la balle se compose surtout des articles de rachis, axes de l'épi, les enveloppes, fragiles, disparaissant le plus souvent lors de la carbonisation 


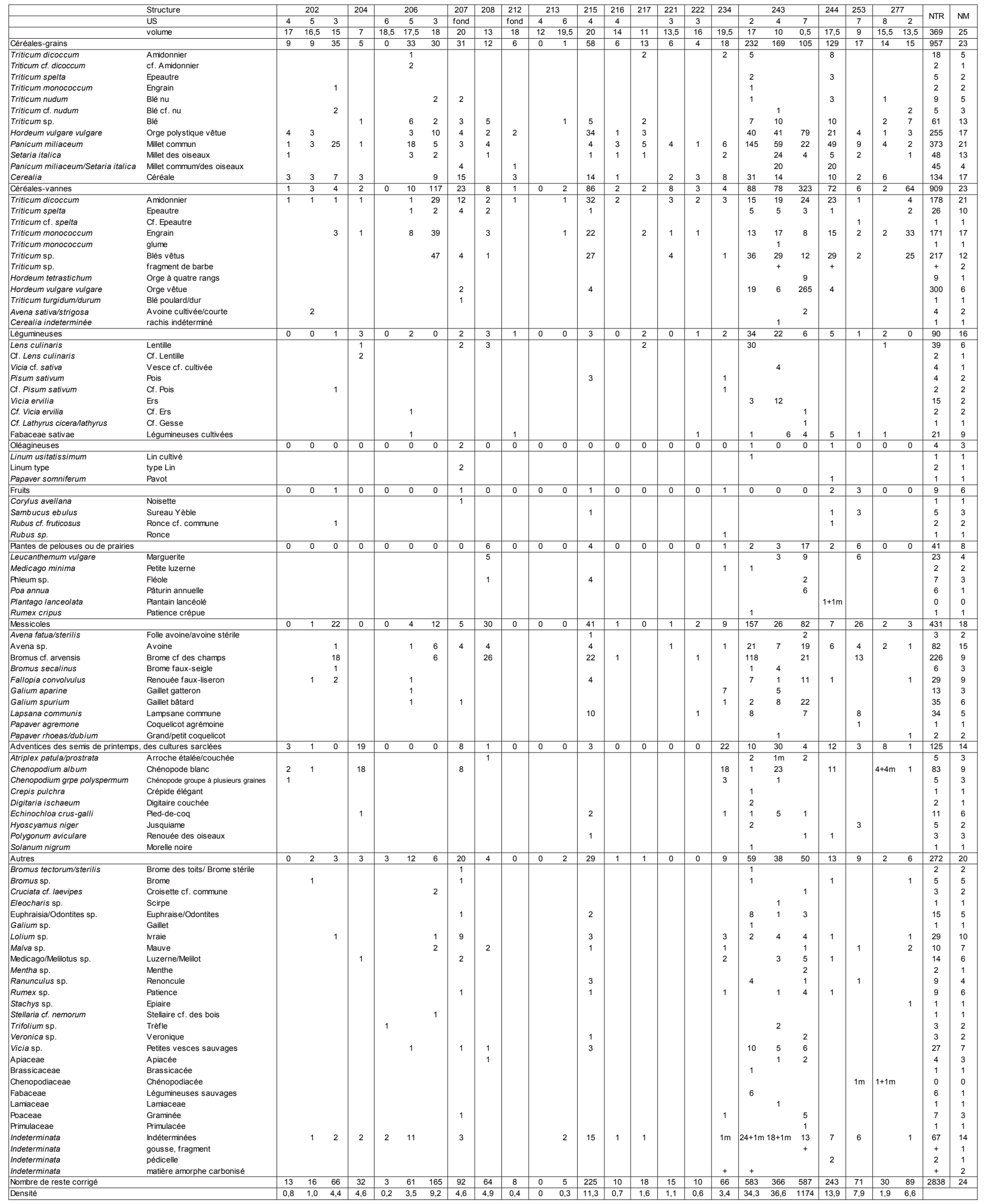

Tableau 3 : Décompte des carporestes collectés aux Rogelins, exprimé en nombre de restes corrigé. Tableau F. Durand.

Table 3: Results of the archaeobotanical samples from the Rogelins (in CNR: corrected number of remains, numbers of fragments are divided to provide estimation for entire remains). 
Tableau 4 : Composition des assemblages carpologiques de densités moyennes à fortes. Le rapport balle/grain ou rachis/grain sont calculés à partir du NRC (cf. compris; cf. identification incertaine) et les pourcentages à partir du type de restes le plus abondant. Les résultats supérieurs à 1 traduisent la présence de déchets de nettoyage des récoltes, à l'inverse les rapports nettement inférieurs à 1 indiquent la présence de grains nettoyés. Tableau F. Durand. Table 4: Carpological assemblages from samples of average and high density. Ratio of spikelet baselgrain or rachis internodelgrain based on CNR (including cf.; cf. uncertain identification), percentages are calculated from the most abundant types of remains. Values greater than 1 , the results of the ratios reflect dehusking wastes whereas for figures significantly less than 1 ratio indicate that assemblages come from cleaned crop.

\begin{tabular}{|c|c|c|c|c|c|}
\hline & St 215 & St 244 & St 243 US 2 & St 243 US 4 & St 243 US 7 \\
\hline NRC & 225 & 243 & 583 & 366 & 587 \\
\hline Densité & 11,3 & 13,9 & 34,3 & 36,6 & 1174 \\
\hline NRC céréales & 144 & 201 & 343 & 274 & 454 \\
\hline \multicolumn{6}{|l|}{ Amidonnier (épillet $=2$ grains pour 1 base) } \\
\hline NRC grain & & 8 & 5 & & \\
\hline NRC balle & 32 & 23 & 15 & 19 & 24 \\
\hline rapport balle/grain $=N R C$ b alle $* 2 / N R C$ grain & & 6 & 6 & & \\
\hline \multicolumn{6}{|l|}{ Epeautre (épillet $=2$ grains pour 1 base) } \\
\hline NRC grain & & 3 & 2 & & \\
\hline NRC balle & 1 & 1 & 5 & 5 & 3 \\
\hline rapport balle/grain $=N R C$ b alle $* 2 / N R C$ grain & & 0,67 & 5 & & \\
\hline \multicolumn{6}{|l|}{ Engrain (épillet $=1$ grain pour 1 base) } \\
\hline NRC grain & & & 1 & & \\
\hline NRC balle & 22 & 15 & 13 & 17 & 8 \\
\hline rapport balle/grain $=N R C$ balle $/ N R C$ grain & & & 26 & & \\
\hline \multicolumn{6}{|c|}{ Blés non identifiés (on retiendra en moyenne 1,5 grain pour une base) } \\
\hline NRC grain & 5 & 10 & 7 & 10 & \\
\hline NRC balle & 27 & 29 & 36 & 29 & 12 \\
\hline rapport vanne/grain $=N R C$ balle $e^{*} 1,5 / N R C$ grain & 8,10 & 4,35 & 7,71 & 4,35 & \\
\hline$\%$ blés vêtus & 56,94 & 33,83 & 20,12 & 25,55 & 10,35 \\
\hline$\%$ blés nus & 0,00 & 1,49 & 0,29 & 0,36 & 0,00 \\
\hline$\%$ orge (un article de rachis porte 3 grains) & 23,61 & 10,45 & 11,66 & 14,96 & 60,35 \\
\hline nbre grain & 34 & 21 & 40 & 41 & 79 \\
\hline nbre de rachis & 4 & 4 & 19 & 6 & 274 \\
\hline rapport vanne/grain $=N R C$ rachis $* 3 / N R C$ grain & 0,35 & 0,57 & 1,43 & 0,44 & 10,41 \\
\hline$\%$ millets & 3,47 & 36,82 & 42,27 & 37,59 & 5,73 \\
\hline$\%$ légumineuses & 1,20 & 1,91 & 5,61 & 5,59 & 23,00 \\
\hline$\%$ messicoles & 18,22 & 2,88 & 26,93 & 7,10 & 13,97 \\
\hline$\%$ adventices des cultures de printemps ou sarclées & 1,33 & 4,94 & 1,72 & 8,20 & 0,68 \\
\hline$\%$ prairies ou pelouses & 1,78 & 0,82 & 0,34 & 0,82 & 2,90 \\
\hline
\end{tabular}
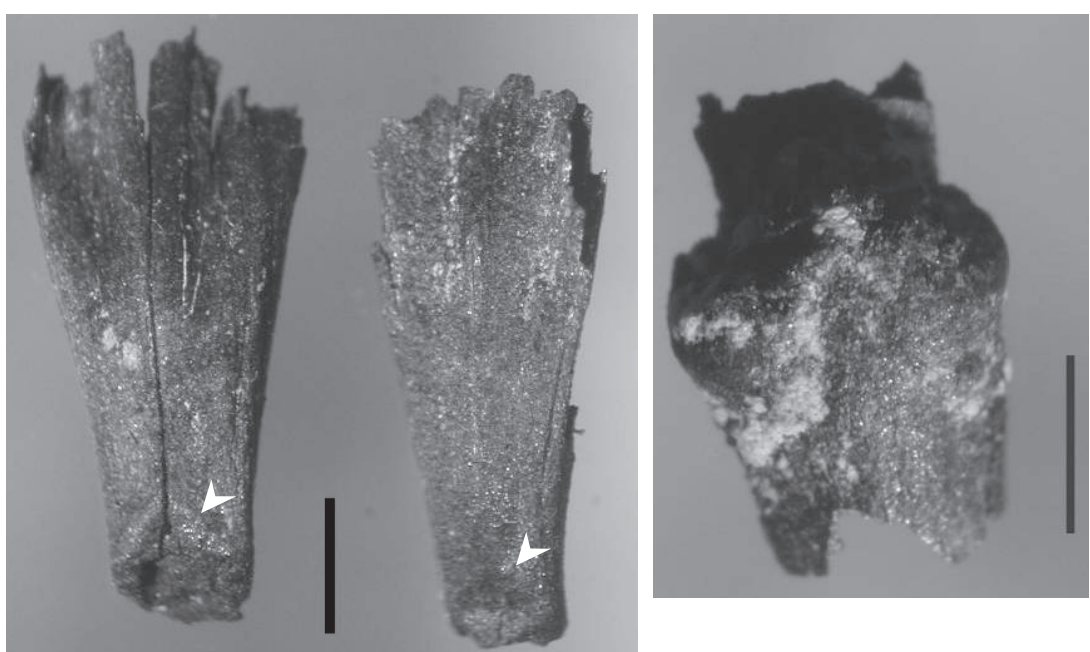

Figure 25 : Exemples de carporestes découverts aux Rogelins. À gauche, bases de lemme d'orge à quatre rangs, les flèches blanches marquent les stigmates en forme de fers à cheval caractéristiques. À droite, article de rachis de blé dur ou de blé poulard. Clichés F. Durand.

Figure 25: Charred remains from the Rogelins. On left, lemma bases of four row Barley, the white arrows indicate the typical horseshoe-shaped depression. On the right, rachis internode of rivet/macaroni wheat. déjà signalée dans le Loiret par B. Pradat (2010) au Bronze final IIIb.

Trois autres céréales apparaissent moins fréquemment, elles sont considérées comme des cultures secondaires. Le millet des oiseaux est toujours associé au millet commun, mais en moindres proportions. S'il est possible que ces deux céréales soient cultivées en méture, le millet des oiseaux pourrait également être une adventice du millet commun (Marinval, 1995). L'épeautre est également identifié, ainsi que les blés nus, vraisemblablement blé dur ou blé poulard comme l'indique un article de rachis provenant de la fosse 207. Bien que les blés nus appartiennent certainement à des cultures secondaires, il est possible que leur importance soit sous-estimée du fait de la nature des assemblages. En effet, le nettoyage des récoltes de blés vêtus engendre une quantité plus importante de déchets, et leur traitement en fonction des besoins multiplie leurs chances de découverte, aux dépends des céréales nues.

Le statut de l'avoine est généralement difficile à définir $\mathrm{du}$ fait des problèmes d'identification au rang spécifique. L'identification à l'espèce n'est possible qu'à partir des bases de lemmes souvent absentes dans les échantillons archéologiques. À Chacé, ces éléments ont été retrouvés et ils révèlent la présence conjointe de la forme domestique (Avena sativa/strigosa) et de la forme sauvage (Avena fatual sterilis). Pourtant l'identification de la forme domestique n'atteste pas à elle seule de la mise en culture de l'avoine puisqu'elle se comporte parfois comme une adventice des autres céréales. Prenant en considération le taux de brome des champs et de brome faux-seigle, d'autres messicoles 
fréquentes, afin de pondérer le taux avoine et de tenter de détecter si ses attestations traduisent ou non une culture de la céréale à Chacé (Ruas et Zech-Matterne, 2012), il apparaît que le pourcentage de grains d'avoine est bien inférieur à celui des bromes. Ce résultat indiquerait que les deux formes d'avoine sont des adventices. Néanmoins, considérant que la fréquence d'attestation des grains d'avoine (15 mentions) est bien supérieure à celle des bromes (10 mentions), inciterait à considérer l'avoine comme une céréale cultivée. Au vu de ces données, il semble bien délicat de trancher, d'autant plus que l'occupation du site de Chacé s'inscrit probablement dans une phase légèrement antérieure à celle des premiers signes de mise en culture, pendant les $\mathrm{IV}^{\mathrm{e}}$-III ${ }^{\mathrm{e}}$ siècles av. J.-C. (Ruas et Zech-Matterne, 2012).

Les résultats dévoilent une céréaliculture diversifiée reposant sur sept espèces, qui rappelle celle mise en évidence en Touraine et dans le Loiret (Pradat, 2010; 2013) où les mêmes espèces constituent les cultures principales et où l'épeautre et les blés nus sont aussi discrets.

Si les assemblages laissent une large place aux céréales, quelques légumineuses sont également attestées. Parmi elles, figurent la lentille et l'ers, légumineuses les plus fréquentes sur le site. Le pois, la gesse et peut-être la vesce cultivée sont aussi attestées. Comme pour les céréales, ces résultats font écho aux données du Loiret, où la lentille et l'ers sont prépondérants. Les autres légumineuses sont minoritaires et la gesse anecdotique (Pradat, 2010).

Du fait de la nature des assemblages et d'un mode de conservation par carbonisation majoritaire, les oléagineuses et les fruits potentiellement cueillis sont faiblement représentés. Leur culture ou leur cueillette sont toutefois envisagées grâce, pour les oléagineuses, à la présence du lin (F. 243, Us 2, et peut-être dans la fosse 207) et du pavot (F. 244). Les fragments de coquille de noisette et des paléosemences de mûre commune plaident pour une pratique de la cueillette. Parallèlement des graines de sureau yèble sont identifiées. Dans son traité de toxicologie, C.-P. Galtier (1855) évoque des intoxications relatives à l'ingestion cru de baies ou feuilles de ce sureau. La cuisson des baies pourrait peut-être en éliminer les toxines comme c'est le cas pour les tanins des glands (Couplan, 1989). L'auteur rapporte en outre que mélangées aux récoltes les feuilles de sureau yèble éloignent les rongeurs et les insectes et que la plante possède des propriétés tinctoriales. La fréquence de cette espèce sur les sites archéologiques est-elle liée à l'une ou l'autre de ces propriétés?

Pour conclure, la nature des assemblages carpologiques du site de Chacé laissent une large place aux céréales. Seuls quelques indices sont recueillis sur les autres productions agricoles (légumineuses, oléagineuses) et la cueillette. La céréaliculture est basée sur une diversité d'espèces, certaines rustiques comme l'orge vêtue, l'engrain et l'épeautre ou plus productives comme l'amidonnier et les blés nus, des céréales de culture d'hiver ou d'été (millets). Cette diversité, associée à une activité de décorticage régulièrement menée, témoigne d'une agriculture de subsistance dont le but est d'assurer les besoins alimentaires des occupants. Ce type d'agriculture, opposée à une agriculture dont le but est le dégagement de surplus, semble constituer la base sociétale pendant la Protohistoire, sur l'ensemble du territoire français et cela, au moins jusqu'au milieu du second Âge du Fer (Bouby, 2014; Cabanis et Messenier-Jouanet, 2010; Pradat, 2010; 2013; Matterne, 2001; Wiethold, 2010).

Les résultats s'intègrent également bien aux bilans dressés par B. Pradat en Touraine et dans le Loiret. Un ensemble agricole homogène se dessine dans le Centre-Ouest de la France pendant le premier Âge du Fer et La Tène ancienne.

\section{ConClusion}

La fouille des Rogelins a permis l'étude d'une partie d'un habitat daté de La Tène A2/B1, période mal connue sur le plan régional. Elle permet de constater, comme dans d'autres régions d'ailleurs, que la culture matérielle laténienne ne s'individualise clairement qu'à cette époque. Pour la phase antérieure, La Tène A1, l'empreinte hallstattienne est, en effet, encore bien marquée et l'on hésite souvent à classer le mobilier au Hallstatt D3 ou à La Tène A1, on pourrait même penser à imaginer un " Hallstatt D4 ".

La densité des silos et leur capacité de stockage semblent indiquer les réserves d'un habitat dont les bâtiments ne se sont peut-être pas conservés. La communauté résidant aux Rogelins était probablement orientée vers une agriculture de subsistance, comme en témoignent les productions végétales dominées par les céréales, et la gestion des cheptels caractérisés une nouvelle fois par la place prédominante du bœuf dans l'ouest de la Gaule.

\section{Bibliographie}

Auxiette G., Baudry A. et Méniel P., 2010 - « Une histoire de l'élevage dans l'ouest de la Normandie : les sites de Mondeville, Ifs, Fleury, Creully et les autres... ", in Barral P., Dedet B., Delrieu F., Giraud P., Le Goff I., Marion S. et Villard-Le Tiec A. (dir.). L'Âge du fer en Basse-Normandie. Actes du XXXIII' colloque international de l'AFEAF (Caen, 20-24 mai 2009). Annales littéraires, 883, Série Environnement, sociétés et archéologie, 14, Besançon, Presses universitaires de FrancheComté, p. 185-202. 
Barbier S., 1995 - La Croix-Boizard à Brion (Maine-et-Loire), Nantes, Association pour les Fouilles archéologiques Nationales.

BARONE R., 1976 - Anatomie comparée des mammiferes domestiques, Paris, Vigot.

BAUdry A., 2012 - Ressources animales et alimentation carnée à l'Âge du Fer : le cas du nord ouest de la France (Bretagne et BasseNormandie), Thèse de doctorat, Université de Rennes 1, France.

Becze-Deak J., Langohr R. et Verrecchia E.P., 1997 - «Small scale secondary $\mathrm{CaCO} 3$ accumulations in selected sections of the European loess belt. Morphological forms and potential for paleoenvironmental reconstruction ", Geoderma, 76, p. 221252.

BeIJERINCK W., 1976 - Zadenatlas der nederlandsche Flora, ten behoeve van de botanie, palaeontologie, bodemkultuur en warenkennis, Mededeeling vnhet Biologisch Station te Wijster. Drenthe, 30, réédition Backhuys \& Meesters, Amsterdam.

Bellanger P., Cornec J., Forré Ph., Germinet D. et Pirault L., 2014 - "L'établissement gallo-romain du Pontreau à Beauvoir-sur-Mer (Vendée) : un cas d'occupation ancienne dans le marais de Monts ", Revue Archéologique de l'Ouest, 31, p. 289-313.

Bonnabel L., 2010 - "Dépôt de corps humains en structures réutilisées (ou détournées?) durant la protohistoire en Champagne-Ardennes : approche comparative avec les sépultures et éléments d'interprétation ", in BARRAY L. et Boulestin B. (dir.). Morts anormaux et sépultures bizarres. Les dépôts humains en fosses circulaires et en silos du Néolithique à l'âge du Fer, Dijon, Presses Universitaires de Dijon, coll. «Art, Archéologie et Patrimoine ", p. 99-112.

Bouby L., 2003 - "Éclairages carpologiques sur les opérations de traitement des céréales à l'Âge du Bronze dans le sud de la France ", in ANDERson P.C. (dir.), Le traitement des récoltes. Un regard sur la diversité, du Néolithique au présent. Actes des XXIII ${ }^{\mathrm{e}}$ rencontres internationales d'archéologie et d'histoire d'Antibes. APDCA, Antibes, p. 21-46.

Bouby L., 2014 - L'agriculture dans le bassin du Rhône du Bronze final à l'Antiquité. Archives d'Écologie préhistorique, Toulouse.

Boulestin B. et Duday H., 1997 - "Les restes humains ", in Brunaux J.-L. et Méniel P. (dir.), La résidence aristocratique de Montmartin (Oise) du IIt au II s. av. J.-C., Paris, Maison des Sciences de l'Homme, coll. "Documents d'Archéologie Française; 64 », p. 61-173 et 202-206.

Boulestin B. et Duday H., 2012 - «Acquisition, préparation et autres traitements de la tête chez les Gaulois : aspects anthropologiques ", in Boulestin B. et Henry Gambier D. (dir.), Crânes trophées, crânes d'ancêtres et autres pratiques autour de la tête : problèmes d'interprétation en archéologie. Actes de la tableronde plurisciplinaire. BAR International Series 2415. Musée National de Préhistoire, Les Eyzies-de-Tayac (Dordogne, France), 14-16 octobre 2010, p. 139-157.
Bouvet J.-Ph., 1987 - Les Âges du Fer dans le département du Maine-et-Loire, Mémoire de maîtrise, Université de Paris I, Panthéon-Sorbonne, France.

Buchsenschutz O., Colin A., Gruel K. et Ralston I.B.M., 1993 - "Approche du territoire au second Âge du Fer ", in A. Daubigney (éd.). Fonctionnement social de l'Âge du Fer, opérateurs et hypothèses pour la France. Actes de la table ronde de Lons-le-Saunier, 24-26 octobre 1990. Section d'archéologie de la Société d'Émulation du Jura, Centre Jurassien du Patrimoine, Besançon, p. 247-258.

Cabanis M., Mennessier-Jouannet C. (dir.), Bouby L., Hajnalova M. et Wiethold J. (collab.), 2011 - « Economie végétale en basse Auvergne à l'Âge du Bronze et au premier Âge du Fer ", in Delhon C., Théry-Parisot I. et Thiébault S. (dir.). Des hommes et des plantes, Exploitation du milieu et gestion des ressources végétales de la Préhistoire à nos jours. Actes des $\mathrm{XXX}^{\mathrm{e}}$ rencontres internationales d'archéologie et d'histoire d'Antibes, 22-24 octobre 2010. APDCA, Antibes, p. 127-144.

Cappers R.T.J., Bekker R.M. et Jans J.E.A., 2006 - Zadenatlas of Netherland, Groningen Archaeological Studies 4, Barkhuis Publishing, Eelde.

Chaix L. et Méniel P., 2001 - Archéozoologie, Les animaux et l'archéologie, Paris, Errance.

Cherel M.-F., 1996 - Ornementation et comparaisons des céramiques armoricaines du deuxième Âge du Fer, Diplôme d'Etudes Approfondies, Université de Paris I/Panthéon-Sorbonne, France.

Couplan F., 1989 - Le régal végétal, Plantes sauvages comestibles, 1, Flers, Éditions Équilibres.

Courty M.-A. et Fédoroff N., 2002 - « Micromorphologie des sols et des sédiments archéologiques ", in Misкovsкy J.-C. (dir.) Géologie de la Préhistoire : Méthodes, Techniques, Paris, Géopré, p. 511-554.

DAIRE M.-Y., 1992 - Les céramiques armoricaines de la fin de l'Âge $d u$ Fer, Travaux du laboratoire d'anthropologie de Rennes 39, Université de Rennes 1, France.

Debord A., Gomez de Soto J. et Sansibilano-Collilieux M., 2000. - « La tombe à épée et Hache du premier Âge du Fer d'Andonne à Villejoubert (Charente, France) ", Archäologisches Korrespondenzblatt, 30, 2, p. 231-250.

Delattre V., 2000 - « De la relégation sociale à l'hypothèse des offrandes : l'exemple des dépôts en silos protohistoriques au confluent Seine-Yonne (Seine-et-Marne) ", Revue Archéologique du Centre de la France, 39, p. 5-30.

Driesch von DEN A., 1975 - « Die Bewertung pathologishanatomischer Veränderungen an vorund frühgestlichen Tierknochen in Archaeozoological Studies ", in Clason A.T. (dir.). Archeozoological Studies, Actes du colloque de Groningen, New York, American Elsevier.

Dron J.-L., Baudry A., Clément-Sauleau S., Gache D., Marcigny C. et Zaour N., 2010. - « Le site de Condé-sur- 
Ifs ", in Barral P., Dedet B., Delrieu F., Giraud P., Le Goff S., Marion I. et Villard-Le Tiec A. (dir.), L’Âge du fer en Basse-Normandie. Actes du XXXIII ${ }^{\mathrm{e}}$ colloque international de l'AFEAF (Caen, 20-24 mai 2009). Annales littéraires, 883, Série Environnement, sociétés et archéologie, 14. Presses universitaires de Franche-Comté, Besançon, p. 129-166.

Ducos P., 1968 - L'origine des animaux domestiques en Palestine. Institut de Préhistoire de l'Université de Bordeaux, Mémoire 6, Bordeaux.

Galtier C.-P., 1855 - Traité de toxicologie. Tome II, poisons organiques et gazeux, Chamerot, Paris.

Gé T., Courty M.-A., Matthews W. el Wattez J., 1994 "Sedimentary Formation Processes of Occupation Surfaces", in Goldberg P., Nash T. et Petraglia M.D. (dir.), Formation Processes in Archaeological Context, Monographs in World Archaeology, 17, Prehistory Press, p. 149-164.

Germinet D., 2009 - Homme et animal dans les fermes du Poitou à la transition de l'Age du Fer à la période romaine au second $\hat{A g e} d u$ Fer en milieu rural, thèse de doctorat, Université de Tours, France.

Giazzon D. (dir.), Baudry-Dautry A., Chanson K. et Charraud F., 2009. - Eterville, les Prés du Vallon, Institut National de Recherches Archéologiques Préventives, CessonSévigné.

Gomez de Soto J., 2005 - " Des images de têtes d'oiseaux de La Tène 1 en Gaule de l'Ouest ", Archäologisches Korrespondenzblatt, 35, p. 493-501.

Gomez De Soto J. (coord.), Lejars T., Ducongé S., Robin K. et SIREIX C., 2007 - «Du milieu du v ${ }^{\mathrm{e}}$ au III ${ }^{\mathrm{e}}$ siècle av. notre ère en Centre-Ouest, Aquitaine septentrionale et ouest du massif central ", in Mennessier-Jounnnet C., Adam A.-M. et Milcent P.-Y. (éd.). La Gaule dans son contexte européen aux $I^{e}$ et III s. av. n. ère, Actes du XXVII ${ }^{e}$ colloque international de l'Association Française pour l'Étude de l'Âge du Fer, ClermontFerrand, 29 mai- ( $^{\text {er }}$ juin 2003, Lattes, Monographies d'archéologie méditerranéenne, p. 69-89.

Gransar F., 2000 - « Le stockage alimentaire sur les établissements ruraux de l'Âge du Fer en France septentrionale : complémentarité des structures et tendances évolutives ", in Marion S. et Blancquaert G. (éd.). Les installations agricoles de l'âge du fer en France septentrionale, Actes du colloque de Paris de l'École Normale Supérieure, 29-30 novembre 1997, Etudes d'histoire et d'archéologie 6. Editions Rue d'Ulm/Presses de l'École Normale Supérieure, Paris, p. 277-297.

Grant A., 1982 - "The use of tooth wear as a guide to the age of domestic ungulates ", in Wilson B. et PAYne S. (eds), Ageing and sexing animal bones from archaeological sites, British Archaeological Reports, 109, p. 91-108.

Green F., 1979 - "Phosphatic mineralization of seed from archaeological sites ", Journal of Archaeological Science 6, p. 279-284.
Hillman G., 1981 - « Reconstructing crop husbrandry practices from charred remains of crops ", in Mercer R. (dir.), Farming Practices in British Prehistory, Edinburg, Edinburg University Press, p. 123-162.

Hillman G., 1984 - «Interpretation of archaeological plant remains: the application of ethnographic models from Turkey ", in van Zeist W. et Casparie W.A. (eds). Plants and ancient man. Study in Palaeoethnobotany. Rotterdam, Balkema, International Workgroup for Palaeoethnobotany, p. 1-41.

Horard-Herbin M.-P., 1997 - L'élevage et les productions animales dans l'économie de la fin du second Âge du Fer. Le village celtique des Arènes à Levroux. Levroux 4, Revue Archéologique du Centre de la France, sup. 12, Tours.

Hurtrelle J., Monchy E., Rossignol P. et Villes A., 1990 Les débuts du second Âge du Fer dans le Nord de la France, (Les Dossiers de Gauheria 1), Gauheria, Lièvin.

Jacobsthal P., 1944 - Early Celtic Art, Oxford, Clarendon Press. JАСОМЕТ S., 2006 - Identification of cereal remains from archaeological sites, $2^{\mathrm{e}}$ édition, Basel, Basel University, IPAS.

Jahier I., Méniel P. et Ozouf J.-C., 2002 - «Une petite ferme de La Tène ancienne au Pré de la Val à Fierville-les-Parcs ", Revue Archéologique de l'Ouest, 19, p. 15-36.

Jahier I. (dir.), Ghesquière E., Le Goff I., Ozouf J.-C., Savary X. et Yvinec J.-H. (collab.), 2011 - L'enceinte des premiers et seconds Âges du Fer de la Fosse Touzé (Courseulles-sur-Mer, Calvados), entre résidence aristocratique et place de collecte monumentale. Maison des Sciences de l'Homme, Documents d'Archéologie Française 104, Archéologie préventive, Paris.

Lамвот B., 1988 - «Les jattes à bord festonné du Bassin Parisien et du Nord de la France ", Bulletin de la Société Archéologique Champenoise 11, p. 31-83.

Le Goff E., 1992. - La céramique de Pouilladou à Prat. Un habitat armoricain de la Tène Ancienne (Côtes-d'Armor), mémoire de maîtrise, Université de Haute-Bretagne, Rennes, France.

Leman-Delerive G., 1973 - "Les coupes à bords ourlés, chronologie et répartition à l'époque de la Tène ", L'Antiquité Classique XLII, p. 407-426.

Lepetz S., 1996 - L'animal dans la société gallo-romaine de la France du Nord, Revue Archéologique de Picardie, nº spécial 12, Amiens.

Lepetz S. et Oueslati T., 2003 - « La consommation de viande dans les villes romaines d'Ile-de-France au $\mathrm{I}^{\text {er }}$ siècle. Les cas de Meaux et de Paris ", Revue Archéologique du Centre de la France 42, p. 41-59.

Levillayer A., Borvon A. et Hunot J.-Y., 2013 - « Du Hallstatt final à La Tène ancienne en Anjou : les batteries de structures de stockage de Luigné et de Cizay-la-Madeleine (Maine-etLoire) ", Revue Archéologique de l'Ouest, 30, p. 113-142.

Maitay C., 2007 - La peinture sur vases aux âges des métaux dans l'Ouest de la France, Rennes, Presses Universitaires de Rennes. 
Maitay C., 2008 - «Entre traditions et innovations. Le premier Âge du Fer ", in Maitay C. (dir.), Feux de Camp. Un site de hauteur: le Camp Allaric. Du Néolithique à l'Âge du Fer, Poitiers, Musée Sainte-Croix, p. 33-36.

Malrain F., Matterne V. et Méniel P., 2002 - Les paysans gaulois, Paris, Errance, coll. «Hespérides ".

Mariën M.-E., 1961 - La période de La Tène en Belgique : le groupe de la Haine, Monographies d'Archéologie Nationale 2, Musées Royaux d'Art et d'Histoire, Bruxelles.

Marinval P., 1995 - «Données carpologiques françaises sur les Millets (Panicum miliaceum L. et Setaria italica) de la Protohistoire au Moyen Age ", in Hörandner E. (dir.), Millet, Hirse, Millet, Grazer Beiträge zur europäischen Ethnologie, Actes du Congrès de d'Aizenay, 18-19 août 1990. Peter Lang, Frankfurt, p. 31-61.

Matterne V., 2001 - Agriculture et alimentation végétale durant l'Âge du Fer et l'époque gallo-romaine en France septentrionale, Montagnac, éd. Monique Mergoil, coll. «Archéologie des plantes et des animaux ".

Menez Y., 1996 - Une ferme de l'Armorique gauloise : le Boisanne à Plouër-sur-Rance (Côtes-d'Armor), Maison des Sciences de l'Homme, Documents d'Archéologie Française 58, Archéologie préventive, Paris.

Menez Y., 2008 - Le Camp de Saint-Symphorien à Paule (Côted'Armor) et les résidences de l'aristocratie du second âge du Fer en France septentrionale, thèse de doctorat, Université de Paris I/ Panthéon Sorbonne, France.

MÉniel P., 1989 - « Des restes humains dans les habitats gaulois ", Les Nouvelles de l'Archéologie, 35, p. 17-19.

Méniel P., Auxiette G., Germinet D., Baudry A. et HorardHerbin M.-P., 2009 - « L'élevage et la chasse sur les établissements ruraux laténiens : approches régionale, chronologique et hiérarchique ", in Bertrand I., Duval A., Gomez De Soto J. et Maguer P. (dir.), Les Gaulois entre Loire et Dordogne. Actes du XXXI ${ }^{\mathrm{e}}$ colloque international de l'Association Française pour l'Étude de l'Âge du Fer, Chauvigny, 17-20 mai 2007. Association des Publications Chauvinoises, Mémoire XXXIV, Chauvigny, p. 413-442.

Meurers-Blake J. et Lüning J., 1992 - "Some aspects and experiments concerning the processing of glume wheats", in Anderson P.C. (dir.), Préhistoire de l'agriculture: nouvelles approches expérimentales et ethnographiques, Monographie du CRA 6, Paris, CNRS éditions, 341-362.

Milcent P.-Y., 2006 - « Premier âge du Fer médio-atlantique et genèse multipolaire des cultures matérielles laténiennes ", in Vitali D. (dir.). La Préhistoire des Celtes, Actes de la table ronde de Bologne, 28-29 mai 2005, Celtes et Gaulois : l'archéologie face à l'histoire. Bibracte 12/2, Glux-en-Glenne, 81-105.

Nicolini G., 1983 - «Trois sépultures de l'Âge du Fer à Mazerolles (Vienne) ", Gallia, 41,1, p. 7-24.
Nillesse O., 2004 - "Le fourreau d'épée à décor d'appliques de la Reculière (Maine-et-Loire) et son contexte ", Antiquités Nationale, 36, p. 215-228.

Nillesse O., 2012 - "Eléments pour une chronologie des établissements ruraux de la fin de l'Âge du fer dans le sud du BasPoitou in Regards sur la chronologie de la fin de l'âge du Fer (III ${ }^{\mathrm{e}} \mathrm{I}^{\mathrm{er}}$ siècle avant J.-C.) en Gaule non méditerranéenne ", in Barral P. et Fichtr S. (dir.). Chronologie de la fin de l'âge du fer (III'-I siècle avant J.-C.) dans l'Est de la France et les régions voisines. Actes de la table ronde tenue à Bibracte, 15-17 octobre 2007, Bibracte 22. Bibracte, Glux-en-Glenne, p. 273-300.

Nillesse O. et Briand F., Manson A.-L. et Vissac C. (collab.), 2014 - «L'agglomération fortifiée de hauteur de la fin du Premier Âge du Fer de Mervent (Vendée) et la typo-chronologie de la céramique du Premier Âge du Fer dans les Pays de la Loire et les Deux-Sèvres ", Aquitania, XXX, p. 61-102.

Nillesse O. et Bryand J.-M., 2000 - ZAC de Beuzon : la Chaine, établissement gaulois à vocation cultuelle : Ecouflant (Maineet-Loire), Association pour les Fouilles Archéologiques Nationales, Nantes.

Pautreau J.-P., 1991 - «Inhumation du premier Âge du Fer à Antran (Vienne) ", Bulletin de la Société Préhistorique Française, 88 , p. $210-224$.

Pautreau J.-P, Mataro i Pladelasala M. et Villard A., 1993 - Civaux Valdivienne II. Les nécropoles protohistoriques et structures néolithiques. Enclos, fosses, structures de combustion. Société de Recherches Archéologiques, Artistiques, Historiques et Scientifique du Pays Chauvinois, Mémoire VII, Poitiers.

PAYne S., 1973 - « Kill-off-Paterns in Sheep and Goats/The mandibule from Asvan Kale ", Anatolian studies, 23, p. 282-303.

Picon M., 1973 - Introduction à l'étude technique des céramiques sigillées de Lezoux, Université de Dijon, Centre de Recherche sur les Techniques Gréco-Romaines (CRTGR 2).

Poirier P., Gomez de Soto J. et Poissonnier B., 2005 "L'occupation de la Tène ancienne de la Renaîtrie (Châtellerault, Vienne). Remarques sur les débuts du second Âge du Fer en Poitou ", Aquitania, XXI, p. 87-121.

Poplin F., 1985 - «Les Gaulois dépecés de Gournay-sur-Aronde », in Brunaux J.-L., Méniel P. et Poplin F., Gournay $I$ : les fouilles sur le sanctuaire et l'oppidum (1975-1984), Numéro spécial de la Revue Archéologique de Picardie, p. 147-164.

Pradat B., 2010 - « L'économie agro-pastorale dans le Loiret à l'Âge du Fer (du Hallstatt ancien à La Tène finale) : synthèse des données carpologiques ", Revue Archéologique du Centre de la France, 49, p. 103-139.

Pradat B., 2013 - «Un premier bilan des données carpologiques à l'âge du Fer en Touraine ", in Krausz S., Colin A., Gruel K., Ralston I. et Dechezleprêtre T. (dir.), L’âge du Fer en Europe : mélanges offerts à Olivier Buchsenschutz, Bordeaux, Ausonius, p. 387-396. 
Rousseau E., 2010 - Pratique et traitement de la tête humaine sur l'ancien territoire de la Gaule au premier millénaire avant notre ère, Thèse de doctorat, Université de Bordeaux 3, France.

Rousseau E., 2012 - "Pratique des têtes coupées chez les Gaulois : les données archéologiques ", in Boulestin B. et Gambier D. Henry (dir.), Crânes trophées, crânes d'ancêtres et autres pratiques autour de la tête : problèmes d'interprétation en archéologie, Actes de la table-ronde plurisciplinaire. BAR International Series 2415. Musée National de Préhistoire, Les Eyzies-de-Tayac (Dordogne, France), 14-16 octobre 2010, p. 117-138.

Ruas M.-P., Zech-Matterne V. et Dietsch-Sellami M.-F., Pradat B. et Preiss S. (collab.), 2012 - « Les avoines dans les productions agro-pastorales du Nord-Ouest de la France. Données carpologiques et indications textuelles ", in Carpentier V., Marcigny C. (dir.). Des hommes aux champs; Pour une archéologie des espaces ruraux du Néolithique au Moyen Âge, Rennes, Presses Universitaires de Rennes, p. 327-365.

SCHMid E., 1972 - Atlas of bones for prehistorians, archaeologists and quaternary geologists, Amsterdam/London/New York, Elsevier Publishing Company.

Schwappach F., 1969 - "Stempelverzierte Keramik von Armorica. Marburger Beiträge zur Archäologie der Kelten », in Herman O. et Habelt R., Festschrift für Wolfgang Dehn zum 60 Geburtstag am 6. Juli 1969, Herausgegeben von OttoHerman Frey. R. Habelt, Bonn, p. 213-287.

Sigaut F., 1981 - «Identification des techniques de conservations et de stockage des grains ", in Sigaut F., Gast M. (dir.), Les techniques de conservations des grains à long terme, tome 2, Paris, CNRS, p. 156-180.

Tela Botanica. De [http://www.tela-botanica.org/site:botanique], consulté en novembre 2015.

Viau Y., PÉan E. et Le Goff E., 1999 - Chanzeaux, la Morinière. Association pour les Fouilles Archéologiques Nationales, Nantes.

Wiethold J., 2010 - Les Trois Domaines, Meuse (55), la Hachie. Macrorestes végétaux carbonisés provenant d'occupations protohistoriques et médiévales, Rapport archéobotanique non publié, Institut National de Recherches Archéologiques Préventives, Metz.

Yvinec J.-H., 1995 - «Étude archéozoologique du site de Brion ", in Barbier S., La Croix Boizard à Brion (Maine-etLoire), Nantes, Association pour les Fouilles Archéologiques Nationales.

Zusammenfassung: Chacé, les Rogelins: ein Fundplatz vom Beginn der Latènezeit im Anjou - Eine vom Institut national de recherches archéologiques preventives (Inrap) durchgeführte Rettungsgrabung bei Chacé „Les Rogelins" (Maine-et-Loire, Frankreich) hat gestattet, eine Siedlung der Latènezeit zu untersuchen, die insbesondere durch zahlreiche Speichergruben charakterisiert ist. Die Analyse des archäologischen Fundmaterials erlaubt es, die Siedlung der Frühlatènezeit zuzuweisen, einer Epoche, die in der Region Pays-de-Loire schlecht dokumentiert ist. Geomorphologische und mikromorphologische Analysen sowie die Untersuchung der Tier-und Menschenknochen sowie der verkohlten pflanzlichen Makroreste haben zur Interpretation des Fundplatzes wichtige Beiträge geliefert.

Resumen: Chacé, les Rogelins: un yacimiento de los inicios de La Tène en Anjou - La excavación llevada a cabo por el Inrap en el municipio de Chacé en el departamento de Maine-et-Loire ha permitido estudiar parte de un poblado de la Segunda Edad del Hierro caracterizado por la presencia de silos. El estudio de los materiales indica una ocupación de la fase antigua de La Tène, periodo mal documentado en la región de Pays de la Loire. Los análisis geomorfológicos, micromorfológicos, el examen de los restos óseos faunisticos y antropológicos y de los restos carpológicos completan la interpretación del yacimiento.

Schlüsselwörter: Frühlatènezeit, offene ländliche Siedlung, Silogruben, Keramik, naturwissenschaftliche Analysen.

Palabras clave: La Tène antigua, poblado abierto, silos, cerámica, análisis. 